\title{
Deep imaging survey of the environment of $\alpha$ Centauri
}

\section{Adaptive optics imaging of $\alpha$ Cen B with VLT-NACO ${ }^{\star}$}

\author{
P. Kervella ${ }^{1}$, F. Thévenin ${ }^{2}$, V. Coudé du Foresto ${ }^{1}$, and F. Mignard ${ }^{2}$ \\ 1 LESIA, UMR 8109, Observatoire de Paris-Meudon, 5 place Jules Janssen, 92195 Meudon Cedex, France \\ e-mail: Pierre.Kervella@obspm.fr \\ 2 Département Cassiopée, UMR 6202, Observatoire de la Côte d'Azur, BP 4229, 06304 Nice Cedex 4, France
}

Received 4 July 2006 / Accepted 17 August 2006

\begin{abstract}
Context. $\alpha$ Centauri is our closest stellar neighbor, at a distance of only $1.3 \mathrm{pc}$, and its two main components have spectral types comparable to the Sun. This is therefore a favorable target for an imaging search for extrasolar planets. Moreover, indications exist that the gravitational mass of $\alpha \mathrm{Cen} \mathrm{B}$ is higher than its modeled mass, the difference being consistent with a substellar companion of a few tens of Jupiter masses.

Aims. We searched for faint comoving companions to $\alpha$ Cen B. As a secondary objective, we built a catalogue of the detected background sources.

Methods. We used the NACO adaptive optics system of the VLT in the $J, H$, and $K_{\mathrm{s}}$ bands to search for companions to $\alpha$ Cen B. This instrument allowed us to achieve a very high sensitivity to point-like sources, with a limiting magnitude of $m_{K \mathrm{~s}} \approx 18$ at $7^{\prime \prime}$ from the star. We complemented this data set with archival coronagraphic images from the HST-ACS instrument to obtain an accurate astrometric calibration.

Results. Over the observed area, we did not detect any comoving companion to $\alpha$ Cen B down to an absolute magnitude of 19-20 in the $H$ and $K_{\mathrm{s}}$ bands. However, we present a catalogue of 252 background objects within about $15^{\prime \prime}$ of the star. This catalogue fills part of the large void area that surrounds $\alpha$ Cen in sky surveys due to the strong diffused light. We also present a model of the diffused light as a function of angular distance for the NACO instrument, that can be used to predict the background level for bright star observations.

Conclusions. According to recent numerical models, the limiting magnitude of our search sets the maximum mass of possible companions to 20-30 times Jupiter, between 7 and $20 \mathrm{AU}$ from $\alpha$ Cen B.
\end{abstract}

Key words. techniques: high angular resolution - stars: individual: $\alpha$ Cen - stars: planetary systems - stars: solar neighbourhood astronomical data bases: miscellaneous - infrared: stars

\section{Introduction}

Our closest stellar neighbor, the $\alpha$ Cen visual triple star $(d=$ $1.34 \mathrm{pc}$ ), is an extremely attractive target for an extra-solar planet search. The main components, $\alpha$ Cen A (HD 128620) and B (HD 128621), are G2V and K1V solar-like stars (e.g. have solarlike asteroseismic oscillation frequencies), while the third member is the red dwarf Proxima (M5.5V). In all imaging planet searches, the main difficulty is in retrieving the planetary signal in the bright point-spread function (hereafter PSF) from the star. The proximity of $\alpha$ Cen is a clear advantage as it allows a faint companion to be easily separated angularly from the star itself down to orbital distances as close as a few astronomical units. After a discussion of the potential for companions around $\alpha$ Cen (Sect. 2), we present our adaptive optics observations (Sect. 3) and the existing data from the HST archive (Sect. 4), followed by the catalogue of the detected sources (Sect. 5) and a discussion (Sect. 7).

$\star$ Table 7 is only available in electronic form at http://www . aanda.org

\section{Why search for companions around $\alpha$ Cen?}

Two factors led us to consider the possibility of a planetary mass companion orbiting around $\alpha$ Cen B: the mass discrepancy between models and the dynamical mass of B on one hand, and the existence of chaotically stable orbits at intermediate distances from the star on the other hand.

\subsection{The mass of $\alpha$ Cen $B$}

Thévenin et al. (2002, hereafter T02) have proposed a model of $\alpha$ Cen B that reproduces well both the asteroseismic observables and the high-precision radius measurement obtained using longbaseline interferometry (Kervella et al. 2003; Bigot et al. 2006). This model yields a mass of $M_{\mathrm{B}}=0.907 \pm 0.006 M_{\odot}$ for $\alpha$ Cen B, in agreement with the study by Guenther \& Demarque (2000). Simultaneously, Pourbaix et al. (2002, hereafter P02) have measured the radial velocity of this star with an overall precision of a few $\mathrm{m} \mathrm{s}^{-1}$ and deduced a dynamical mass of $M_{\mathrm{B}}=0.934 \pm$ $0.006 M_{\odot}$. The difference between the model mass and the dynamical mass of B reaches $\Delta M_{\mathrm{B}}=0.027 \pm 0.008 M_{\odot}=28 \pm$ 9 Jupiter masses (hereafter $M_{\mathrm{J}}$ ). No such difference is observed for $\alpha$ Cen $\mathrm{A}$, for which the agreement is excellent between the 
Table 1. Properties of $\alpha$ Cen A and B.

\begin{tabular}{lcc}
\hline \hline & $\alpha$ Cen A & $\alpha$ Cen B \\
\hline Other names & HD 128620 & HD 128621 \\
& HIP 71683 & HIP 71681 \\
\hline$m_{V}$ & -0.01 & 1.33 \\
$m_{J}$ & -1.16 & -0.01 \\
$m_{H}$ & -1.39 & -0.49 \\
$m_{K}$ & -1.50 & -0.60 \\
Spectral type & $\mathrm{G} 2 \mathrm{~V}$ & $\mathrm{~K} 1 \mathrm{~V}$ \\
$T_{\text {eff }}(\mathrm{K})^{a}$ & $5790 \pm 30$ & $5260 \pm 50$ \\
$\log g^{a}$ & $4.32 \pm 0.05$ & $4.51 \pm 0.08$ \\
{$[\mathrm{Fe} / \mathrm{H}]^{a}$} & $0.20 \pm 0.02$ & $0.23 \pm 0.03$ \\
\hline
\end{tabular}

${ }^{a}$ From Morel et al. (2000) and references therein.

measured properties of $\alpha$ Cen A and the model from T02, computed using the mass measured by $\mathrm{P} 02$.

In order to explain the $3 \sigma$ difference between the modeled and measured mass of $\mathrm{B}$, a possible scenario is that the implicit assumption made by $\mathrm{P} 02$ of a two-body system is incorrect due to the existence of a companion orbiting $\alpha$ Cen $\mathrm{B}$. We note that these authors have introduced a correction of the radial velocity of $B$, as they find an offset with respect to the data obtained by Kamper \& Wesselink (1978). This correction may mask the signature of a long-period, low-mass companion orbiting B. The contribution of Proxima to the radial velocity of the main pair is negligible (due to its large distance from the A-B pair). Alternatively, a companion could also orbit the A-B pair on a very long period orbit and currently be located closer to B. Its gravitational contribution would make B appear heavier in the A-B interaction. This is however less probable, as the mass of this companion would have to be significantly higher than the proposed $\approx 30 M_{\text {J }}$ to compensate for its increased distance.

In summary, an $M \simeq 30 M_{\mathrm{J}}$ brown dwarf (hereafter $\mathrm{BD}$ ) within $\simeq 10^{\prime \prime}$ from $\alpha$ Cen B (or up to $50-100^{\prime \prime}$ if orbiting around the pair) could be a viable explanation for the mass discrepancy between P02 and T02. This hypothesis is also favored by the fact that the $\alpha$ Cen system is metal-rich and $\alpha \mathrm{Cen} \mathrm{A}$ is Li-poor, as expected for stars hosting massive planets (Santos et al. 2003; Israelian et al. 2004).

Table 1 lists the relevant physical properties of $\alpha$ Cen A and $\mathrm{B}$, and the astrometric and orbital parameters of the pair are given in Table 2. The position of the barycenter is computed from the Hipparcos astrometric solution of components A and $\mathrm{B}$ using the masses of Table 2 . This gives a perfect consistency with the Hipparcos data, but with an accuracy limited by the poor astrometric solution of the B component. Another solution would have been to use the astrometric solution of component $\mathrm{A}$ and the orbital elements of the system, whose uncertainty is hard to evaluate. The difference between the two approaches is about 0.02 arcsec, so it can be used as a good estimate of the uncertainty of the astrometric position of the barycenter in the ICRS frame at epoch J 1991.25.

\subsection{Orbital stability}

Presently, at least 15 examples of extrasolar planets are known to orbit binary star members: $16 \mathrm{Cyg} \mathrm{B}, v \mathrm{And}, \tau$ Boo, etc. (Eggenberger et al. 2003; Eggenberger et al. 2004; Mugrauer et al. 2005). The 40 yrs period binary $\gamma$ Cep is also very likely the host of a $1.3 M_{\mathrm{J}}$ planet on a 1.8 AU orbit (Cochran et al. 2002). Wiegert \& Holman (1997) have identified how stable orbits can be found within $2^{\prime \prime}$ of $\alpha$ Cen B (interior planets) or at
Table 2. Astrometric parameters of $\alpha$ Cen A-B.

\begin{tabular}{|c|c|}
\hline 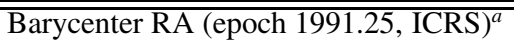 & $14: 39: 40.216$ \\
\hline Barycenter Dec (epoch 1991.25, ICRS $)^{a}$ & $-60: 50: 13.58$ \\
\hline Proper motion RA (mas) & -3642.95 \\
\hline Proper motion Dec (mas) & 694.75 \\
\hline Radial velocity $(\mathrm{km} / \mathrm{s})$ & -20 \\
\hline Galactic long. $\left({ }^{\circ}\right)$ & 315.73 \\
\hline Galactic lat. $\left({ }^{\circ}\right)$ & -0.68 \\
\hline Parallax (mas) ${ }^{b}$ & $747.1 \pm 1.2$ \\
\hline Semi-major axis $a\left({ }^{\prime \prime}\right)^{c}$ & 17.59 \\
\hline Period $P(\mathrm{yr})^{c}$ & 79.9 \\
\hline Excentricity $e^{c}$ & 0.519 \\
\hline Inclination $i\left({ }^{\circ}\right)^{c}$ & 79.23 \\
\hline Long. of ascending node $\Omega\left({ }^{\circ}\right)^{c}$ & 204.82 \\
\hline Longitude of pericenter $\omega\left({ }^{\circ}\right)^{c}$ & 231.80 \\
\hline Reference epoch ${ }^{c}$ & 1955.59 \\
\hline$M_{\mathrm{A}}\left(M_{\odot}\right)^{d}$ & 1.10 \\
\hline$M_{\mathrm{B}}\left(M_{\odot}\right)^{d}$ & 0.91 \\
\hline
\end{tabular}

${ }^{a}$ From Hipparcos (ESA 1997). ${ }^{b}$ Parallax from Söderhjelm (1999). ${ }^{c}$ Orbital elements from Pourbaix (2000). ${ }^{d}$ Masses from Thévenin et al. (2002).

distances of up to $50^{\prime \prime}$ (exterior planets, orbiting the pair). As a further incentive, it has been demonstrated that the Kozai resonance (Holman et al. 1997; Innanen et al. 1997) can prevent the ejection of a binary star companion through chaotic variations in the excentricity of its orbit. This mechanism is invoked by Mazeh et al. (1997) to explain the presence of the planet around 16 Cyg B. High relative inclinations favor this mechanism, and it can also be observed in the Solar system through the secular perturbations introduced by Jupiter on asteroids (Kozai 1962). Such a dynamical, chaotic behavior could stabilize the orbit of a $\mathrm{BD}$ around $\alpha$ Cen B beyond the maximum angular separation found by Wiegert \& Holman (1997). Recently, a hot Jupiter was detected around the primary star of the triple system HD 188753 (Konacki et al. 2005). The semi-major axis of the primary-secondary orbit is $a=12.3 \mathrm{AU}$, only half of $\alpha$ Cen A-B $(a=23.7 \mathrm{AU})$. Moreover, any angular separation can exist for a companion in orbit around the $\alpha$ Cen pair. This is such a favorable target for deep imaging of its environment that it stands out as an important step in testing the results of these numerical simulations.

\section{NACO adaptive optics imaging}

\subsection{Observations}

We have chosen to adapt our observation technique depending on the angular distance to the star. Very close to the two stars, within a radius of about $20^{\prime \prime}$, adaptive optics (subsequently AO) imaging allows us to reach the highest sensitivity thanks to the concentration of the companion light within the Airy disk. The contrast between the companion and the diffused light background is much more favorable than for atmosphere-limited imaging. At distances of more than $20^{\prime \prime}$, the diffused light is less of a problem, and classical (non-AO) imaging is the best solution. Moreover, the degradation of the AO correction quality at such large distances from the star would not bring a significant improvement in the sensitivity. We will present our wide-field imaging observations of the environment of $\alpha$ Cen in a forthcoming paper.

We thus observed the environment of $\alpha$ Cen B using the Nasmyth Adaptive Optics System (NAOS, Rousset et al. 2000; 
Rousset et al. 2003) of the Very Large Telescope (VLT), coupled to the CONICA infrared camera (Lenzen et al. 1998). The combination of these two devices is abbreviated as NACO. NAOS is equipped with a tip-tilt mirror and a deformable mirror controlled by 185 actuators, as well as two wavefront sensors: one for visible light and one for the infrared domain. For our observations, we exclusively used the visible light wavefront sensor. The detector is a $1024 \times 1024$ pixels ALADDIN InSb array. As its name indicates, NACO is installed at the Nasmyth focus of the Unit Telescope 4 (Yepun), the easternmost of the four $8 \mathrm{~m}$ telescopes of the VLT. Our observations were obtained shortly after the recoating of the primary mirror, which was executed in October 2003, in order to benefit from the best possible uniformity in reflectivity. This excellent state of the primary mirror coating allowed us to minimize the PSF light leaks, and consequently to obtain the best sensitivity. The NACO instrument offers two coronagraphic modes, based on a classical Lyot coronagraph or an innovative four-quadrant phase mask (Rouan et al. 2000), but due to the extreme brightness of $\alpha \mathrm{Cen}$, the rejection level was insufficient for preventing the saturation of the detector. As a consequence, we preferred to use the direct imaging mode and keep the two stars outside the detector. This was achieved simply by offsetting the NACO field of view.

The first series of observations were obtained between February 18 and April 10, 2004. We obtained repeated short exposures of four fields arranged in a cross around B and (accidentally) one field East of A using the S13 mode of CONICA and $J H K_{\mathrm{s}}$ broadband filters. The pixel scale in this mode is $13.26 \pm$ $0.03 \mathrm{mas} / \mathrm{pix}$ (Masciadri et al. 2003), giving a field of view of $13.6^{\prime \prime} \times 13.6^{\prime \prime}$. This small scale results in an excellent sampling of the PSF, with $\simeq 5 \mathrm{pix} / \mathrm{PSF}$, an important advantage when distinguishing the point-like sources from the speckle cloud based on their dimension and shape. For all fields, the AO reference star was $\alpha$ Cen B.

We repeated the same observations one year later in order to identify the proper-motion companions, using the $K_{\mathrm{s}}$ filter only because all the sources identified in the $J$ and $H$ bands were also detected in $K_{\mathrm{s}}$. One image of the southern field was obtained in July 2004, but due to operational constraints, the remaining observations were conducted in February-March 2005. All observations were obtained using the Fowler-sampling, highsensitivity mode of CONICA. The individual exposures were limited to $5.0 \mathrm{~s}$ in the $J$ band and $3.5 \mathrm{~s}$ in the $H$ and $K_{\mathrm{s}}$ bands. The complete observation $\log$ is presented in Tables 3-5, together with the seeing ${ }^{1}$ observed in the visible by the DIMM (Sarazin \& Roddier 1990; Martin et al. 2000). Our NACO images were obtained in general under good seeing conditions, many of them at or below the $0.7^{\prime \prime}$ level in the visible (Fig. 1). This is a clear advantage in detecting very faint sources as the coherent energy (encircled in the core of the diffraction-limited PSF) can then rise up to $70 \%$ or more. In each field, the total integration time for each epoch varies between 10 and 20 min. Depending on the location around $\alpha \mathrm{Cen}$, up to four epochs are available. The resulting total coverage in the $J, H$ and $K_{\mathrm{s}}$ bands is presented in Fig. 2. The $H$ and $J$ band data cover a comparatively smaller area and only one epoch was obtained in the $J$ band due to the relatively lower sensitivity compared to $H$ and $K_{\mathrm{s}}$. When only one epoch was obtained, the search for comoving companions is not possible (see also Sect. 6).

\footnotetext{
${ }^{1}$ Available at http://archive.eso.org/asm/ambient-server
}

Table 3. Log of the first series of NACO images.

\begin{tabular}{|c|c|c|c|c|c|c|}
\hline Field & Date & $\mathrm{UT}^{a}$ & $\lambda^{b}$ & $\delta t(\mathrm{~s})^{c}$ & $\theta\left({ }^{\prime \prime}\right)^{d}$ & $\mathrm{AM}^{e}$ \\
\hline West B & $2004-02-18$ & $7: 09$ & $J$ & 360 & 0.48 & 1.38 \\
\hline West B & 2004-02-18 & $7: 15$ & $H$ & 392 & 0.49 & 1.37 \\
\hline West B & 2004-02-18 & $7: 21$ & $K_{\mathrm{s}}$ & 322 & 0.49 & 1.35 \\
\hline South B & 2004-02-18 & $8: 10$ & $J$ & 480 & 0.51 & 1.28 \\
\hline South B & 2004-02-18 & $8: 15$ & $H$ & 525 & 0.56 & 1.28 \\
\hline South B & 2004-02-18 & $8: 20$ & $K_{\mathrm{s}}$ & 518 & 0.51 & 1.27 \\
\hline North B & 2004-02-18 & 9:03 & $J$ & 320 & 0.52 & 1.24 \\
\hline North B & 2004-02-18 & 9:08 & $H$ & 500 & 0.45 & 1.24 \\
\hline North B & 2004-02-18 & $9: 14$ & $K_{\mathrm{s}}$ & 500 & 0.54 & 1.24 \\
\hline East B & 2004-02-19 & $8: 33$ & $J$ & 480 & 1.07 & 1.26 \\
\hline East B & 2004-02-19 & $8: 54$ & $H$ & 525 & 0.92 & 1.25 \\
\hline East B & 2004-02-19 & $8: 59$ & $K_{\mathrm{s}}$ & 518 & 0.75 & 1.24 \\
\hline West B & 2004-02-20 & $6: 47$ & $J$ & 360 & 0.72 & 1.41 \\
\hline West B & 2004-02-20 & $6: 52$ & $H$ & 392 & 0.68 & 1.40 \\
\hline West B & $2004-02-20$ & $6: 59$ & $K_{\mathrm{s}}$ & 322 & 0.86 & 1.38 \\
\hline South B & 2004-02-20 & $7: 39$ & $J$ & 480 & 1.03 & 1.31 \\
\hline South B & $2004-02-20$ & $7: 44$ & $H$ & 525 & 1.03 & 1.30 \\
\hline South B & 2004-02-20 & $7: 50$ & $K_{\mathrm{s}}$ & 518 & 0.85 & 1.30 \\
\hline North B & 2004-02-20 & $8: 38$ & $J$ & 480 & 0.74 & 1.25 \\
\hline North B & 2004 & $8: 43$ & $H$ & 750 & 0.71 & 1.25 \\
\hline North B & 2004-02-20 & $8: 49$ & $K_{\mathrm{s}}$ & 740 & 0.71 & 1.25 \\
\hline East B & $2004-02-26$ & $7: 58$ & $J$ & 160 & 1.62 & 1.26 \\
\hline East B & $2004-02-26$ & $8: 03$ & $H$ & 175 & 1.28 & 1.26 \\
\hline East B & $2004-02-26$ & $8: 08$ & $K_{\mathrm{s}}$ & 175 & 1.53 & 1.26 \\
\hline East A & 2004-03-12 & $7: 59$ & $H$ & 175 & 1.37 & 1.24 \\
\hline East A & 2004-03-12 & $8: 02$ & $J$ & 320 & 1.35 & 1.24 \\
\hline East A & 2004-03-12 & 8:04 & $K_{\mathrm{s}}$ & 175 & 1.35 & 1.24 \\
\hline East B & 2004-04-10 & $5: 14$ & $J$ & 480 & 0.61 & 1.26 \\
\hline East B & 2004-04-10 & $5: 23$ & $H$ & 525 & 0.58 & 1.25 \\
\hline East B & 2004-04-10 & $5: 29$ & $K_{\mathrm{s}}$ & 518 & 0.57 & 1.25 \\
\hline
\end{tabular}

${ }^{a}$ Average time of the observation sequence. ${ }^{b}$ Selected broadband filter. ${ }^{c}$ Total exposure time. ${ }^{d}$ DIMM seeing measured in the visible $(\lambda=$ $0.5 \mu \mathrm{m}) .{ }^{e}$ Airmass.

Table 4. Second series of NACO images.

\begin{tabular}{lcclccc}
\hline \hline Field & Date & UT & $\lambda$ & $\delta t(\mathrm{~s})$ & $\theta\left({ }^{\prime \prime}\right)$ & AM \\
\hline South B & $2004-07-25$ & $1: 29$ & $K_{\mathrm{s}}$ & 1008 & 0.99 & 1.37 \\
West B & $2005-02-07$ & $7: 24$ & $K_{\mathrm{s}}$ & 535.5 & 1.10 & 1.44 \\
West B & $2005-02-09$ & $8: 22$ & $K_{\mathrm{s}}$ & 535.5 & 1.05 & 1.31 \\
South B & $2005-02-09$ & $8: 50$ & $K_{\mathrm{s}}$ & 756 & 1.27 & 1.27 \\
West B & $2005-02-09$ & $9: 46$ & $K_{\mathrm{s}}$ & 535.5 & 0.95 & 1.24 \\
East B & $2005-03-28$ & $5: 21$ & $K_{\mathrm{s}}$ & 567 & 0.63 & 1.30 \\
South B & $2005-03-29$ & $4: 04$ & $K_{\mathrm{s}}$ & 756 & 0.87 & 1.45 \\
North B & $2005-03-29$ & $4: 31$ & $K_{\mathrm{s}}$ & 216 & 0.76 & 1.38 \\
\hline
\end{tabular}

Table 5. Third series of NACO images.

\begin{tabular}{lcclccc}
\hline \hline Field & Date & UT & $\lambda$ & $\delta t(\mathrm{~s})$ & $\theta\left({ }^{\prime \prime}\right)$ & AM \\
\hline West B & $2005-07-13$ & $23: 30$ & $K_{\mathrm{s}}$ & 787.5 & 0.75 & 1.24 \\
West B & $2005-07-13$ & $23: 53$ & $H$ & 756 & 0.82 & 1.24 \\
\hline
\end{tabular}

\subsection{Data processing}

The processing was achieved using the IRAF package (v.2.12). The images obtained on each night were dark-subtracted and flat-fielded with standard infrared astronomical techniques. No sky subtraction was done, as the inhomogeneous diffused light from $\alpha$ Cen largely dominates the sky background level, even in the $K_{\mathrm{s}}$ band (see Sect. 3.4). We interpolated the bad pixels and mosaicked the dither pattern using the bright sources visible in each field as references. The observed drift rate remains below 30 mas $^{-1}$ for all fields (less than 2.5 pixels, or half the 


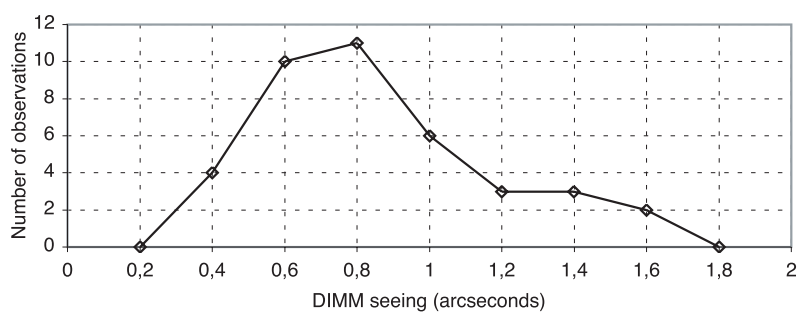

Fig. 1. Histogram of the number of NACO images as a function of DIMM seeing (in visible light, with seeing bins of $0.2^{\prime \prime}$ ).

FWHM of the PSF). This is a remarkable performance for such a large and massive instrument and telescope configuration. For the northern field in the $J$ band, no source was detected in each individual frame, so the registration was achieved using the offsets measured on the $H$ and $K_{\mathrm{s}}$ band images. This procedure is justified by the fact that the observed relative drifts were identical in the three bands. In any case, no source was detected in the northern field in the combined $J$ band image. Bright artefacts ("ghosts") are present in the images of the fields located to the west of both stars. They are probably caused by reflections and interferences in the semi-reflective beam splitters used to separate the visible light (used for wavefront sensing) from the infrared.

\subsection{Astrometric calibration}

The astrometric referencing of narrow-field NACO images of a fast-moving source such as $\alpha$ Cen $\left(\approx 4^{\prime \prime} \mathrm{yr}^{-1}\right)$ is not a straightforward task. It is made all the more difficult as all direct images of the pair are heavily saturated. As a consequence, there are no astrometric reference stars sufficiently close to the pair to attach the NACO images to a solid astrometric reference.

We thus based our absolute astrometric calibration on the computed positions of the $\alpha$ Cen B star using the initial Hipparcos position at J1991.25 to determine the ICRS astrometric position of the barycenter. Then the positions of $\alpha$ Cen B are computed at any other epoch with the orbital elements from Pourbaix (2000) and proper motion measurements from the Hipparcos satellite (ESA 1997). These positions take into account the combined effect of the parallactic apparent displacement, proper motion, and orbital motion of the pair. It should be noted that, even though the resulting apparent displacement is particularly complicated, the accuracy of the available astrometric elements is such that they do not limit the astrometric calibration of our images. As an illustration of the complexity of the apparent motion of $\alpha$ Cen A and B, Fig. 4 shows the ICRS positions of the two stars on the sky for the period 1999-2010.

To transfer the reference coordinates of $\alpha \mathrm{Cen} \mathrm{B}$ to the detected background sources, we used as an intermediate step the HST-ACS coronagraphic images publicly available from the ST-ECF archive facility. These images present the advantage of simultaneously showing an attenuated image of the occulted $\alpha$ Cen $\mathrm{B}$, as well as several background sources that are detectable on our NACO images. We chose the brightest of these sources, which is also the nearest to $\alpha \mathrm{Cen} \mathrm{B}$, numbered 167 in our catalogue (Table 7). This ACS image was obtained on 15 June 2004, for which date we computed the ICRS position of $\mathrm{B}$ to be

$\alpha(\mathrm{B})=14: 39: 32.953 \quad \delta(\mathrm{B})=-60: 50: 08.65$,

thus giving the following coordinates for star \#167:

$\alpha(\# 167)=14: 39: 33.567 \quad \delta(\# 167)=-60: 50: 11.57$.
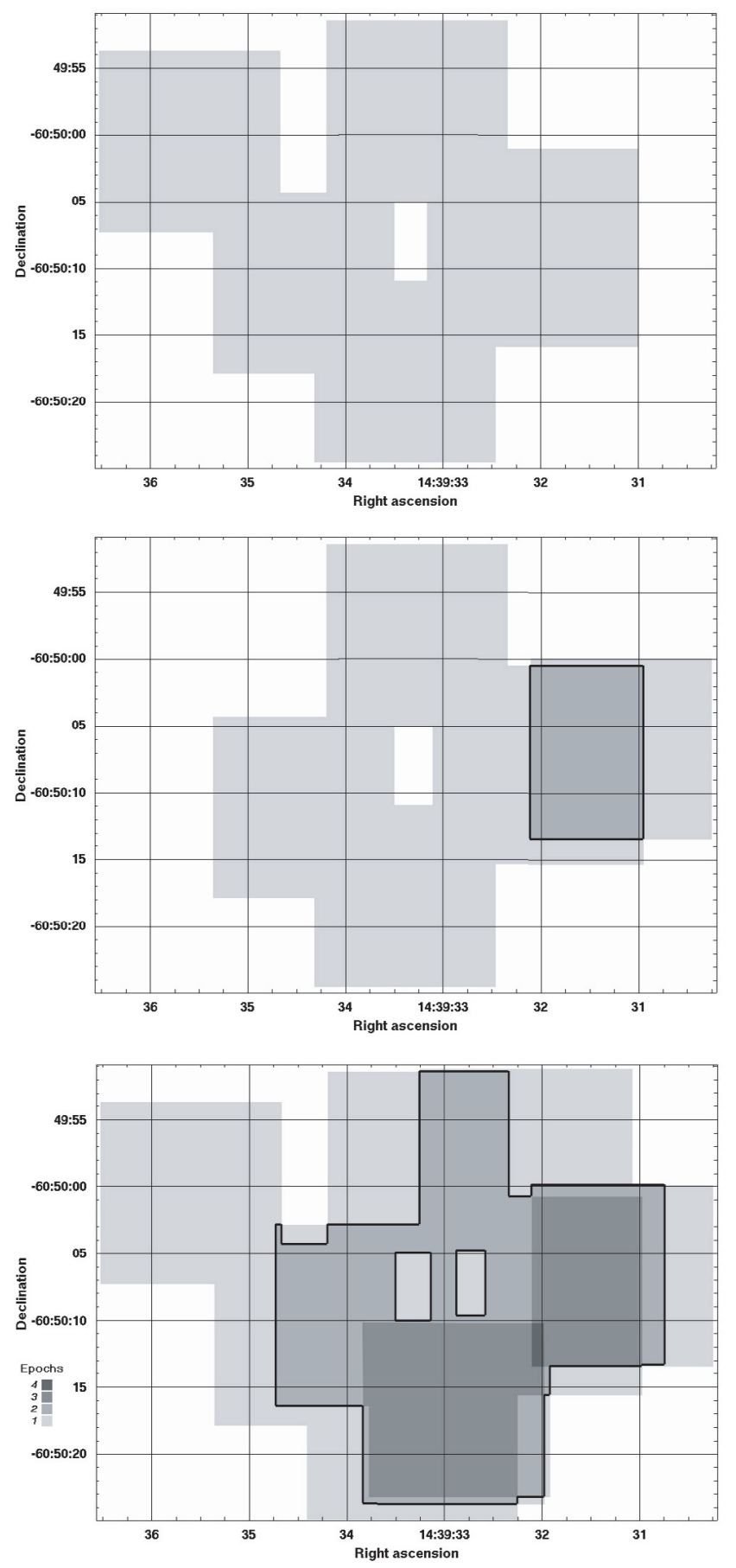

Fig. 2. Number of NACO epochs available for the field around $\alpha$ Cen B in the $J$ (top), $H$ (middle), and $K_{\mathrm{s}}$ (bottom) bands. The grey scale (bottom left) indicates how many epochs were obtained at each position. The thick black line encompasses the domain that was observed at least on two epochs.

Knowing the absolute sky coordinates of each star, a "world coordinate system" (wcs) was defined for each image based on the measured position, the pixel scale of the camera, and the orientation of the field of view ( $y$ axis aligned along the north-south direction). The relative astrometric accuracy over the HST-ACS field is better than 5 mas, therefore introducing a negligible uncertainty in the coordinate transfer.

We would like to stress here that the wcs used for all our images is linked to the computed position of $\alpha$ Cen B on 15 June 2004. Any modification of the computed astrometric 


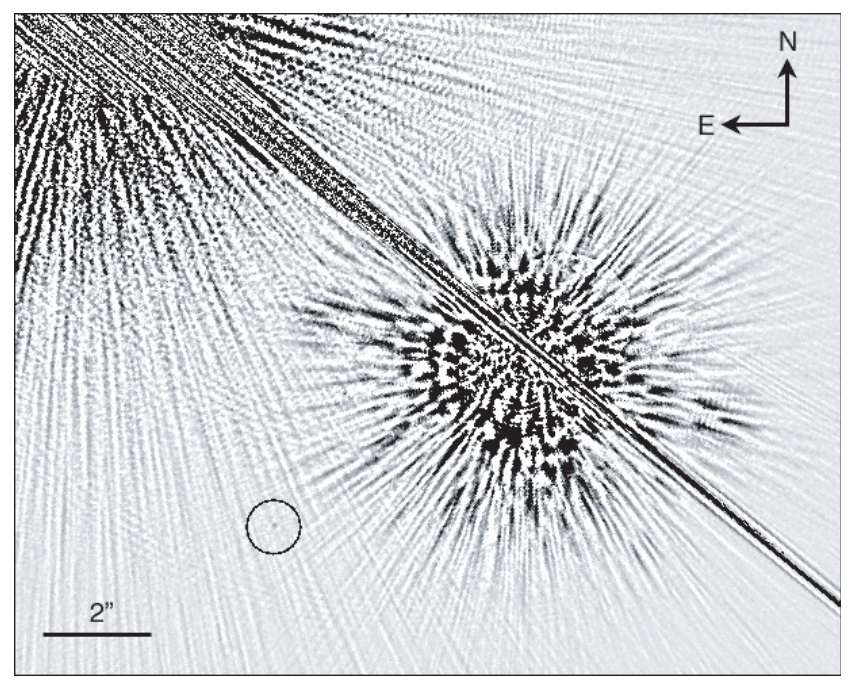

Fig. 3. Extract of an HST-ACS coronagraphic image of $\alpha$ Cen B showing the bright background star \#167 (circled) that was used to transfer the reference astrometric coordinates of $\alpha$ Cen B to the NACO images. The saturated source in the upper left corner is $\alpha$ Cen A.

position of $B$ for this date can be transferred to the source catalogue using a simple translation. Given the small size of the field, we expect an absolute astrometric accuracy better than $\pm 0.10^{\prime \prime}$ from this very simple astrometric reduction. However, the relative position accuracy of the different sources within the same NACO field is much better, with an estimated $\pm 0.03^{\prime \prime}$ ( 2 pixels). The orientation of true north of the S13 camera of NACO was found to be extremely stable and accurate by Chauvin et al. (2005), with an undetectable deviation of less than $0.1^{\circ}$ from the true north-south direction over a period of more than one year (Nov. 2002-Mar. 2004). The uncertainty of the scale is estimated to be less than $0.2 \%$, giving at most one pixel over a field of 30 arcsec.

\subsection{Diffused light}

The main limitation to the sensitivity of imaging close to bright sources is caused by diffused light. It is mostly created inside the telescope and the instrument by imperfect optics and baffling. For the preparation of adaptive optics observations of bright sources, it is important to know the properties of the diffused light to prevent saturation of the detector.

To study its profile in our images, we considered the field located south of $\alpha$ Cen B, thereby avoiding the contamination by the light from $\alpha$ Cen A, which is particularly strong in the northern and eastern fields. A difficulty in measuring the diffused light is that a number of artefacts create local biases. For instance, the large spikes produced by the secondary spider and the ghost reflections visible in the western images should not be included in the background estimation. We thus sampled the background level manually to avoid these artefacts. The result was a series of $\approx 500$ samples $N(\theta)$ in each band, with $\theta$ the angular distance from $\alpha$ Cen B and $N$ the camera counts (in ADUs). These measurements were then converted to magnitudes per squared arcsec taking into account the exposure time ( $\delta t=5.0 \mathrm{~s}$ in $J, 3.5 \mathrm{~s}$ in $H$ and $\left.K_{\mathrm{s}}\right)$, the pixel size $\left(\delta \theta=0.01326^{\prime \prime}\right)$, and the photometric zero point for the night $\left(\mathrm{ZP}_{J}=23.95, \mathrm{ZP}_{H}=23.85, \mathrm{ZP}_{K \mathrm{~s}}=22.95\right)$. In order to obtain a calibrated model that can be applied to other sources, we normalized the resulting magnitudes to a zero magnitude source using the apparent magnitudes $m_{\lambda}(\mathrm{B})$ of $\alpha$ Cen B listed in Table 1. The expression of the measured sky-backgound contrast (in mag $\operatorname{arcsec}^{-2}$ ) is therefore:

$\Delta m_{\lambda}(\theta)=-2.5 \log \left[\frac{N(\theta)}{\delta t \delta \theta^{2}}\right]+\mathrm{ZP}_{\lambda}-m_{\lambda}(\mathrm{B})$.

We subsequently computed a least-square fit to our data using an exponential model of the form:

$\Delta m_{\lambda}(\theta)=a \exp (b \theta)+c$.

As shown in Fig. 5, this type of model is a good match to the observed distribution. The resulting best-fit values of the $(a, b, c)$ coefficients are listed for each band in Table 6. The profiles obtained in the three bands are very similar, and they show a relative flux level with respect to the central source of $\Delta m \approx 9 \mathrm{mag} \operatorname{arcsec}^{-2}$ at a distance of $3^{\prime \prime}$. To extend our diffused background model closer to the central star, we took advantage of the unsaturated acquisition images (left part of Fig. 5). It is interesting to note that the estimated brightness at a large distance from the star tends asymptotically to $m_{K} \approx 13 \mathrm{mag} \mathrm{arcsec}^{-2}$, which is close to the typical Paranal sky brightness level in the $K$ band.

\subsection{Source extraction and photometry}

The greatest difficulty in extracting sources from the diffused light of $\alpha$ Cen is to separate the background inhomogeneities from the true point-like sources. We first high-pass-filtered the combined images using the ring median filter of IRAF (Secker 1995). By adjusting the ring radius precisely to the radius of the PSF, it is possible to isolate the smooth, low spatial frequency diffused light and remove it from the image. This filtering allows a much more robust identification of the point-like sources. With only 252 sources in total, a visual identification was found to be more efficient than an automated detection algorithm. We used the $K_{\mathrm{s}}$ band images for this identification, as all sources detected in $J$ and $H$ were also detected in this band.

The difficulty with automated source identification is to adapt the sensitivity to the rapidly changing background level depending on the distance to the star. The identification of the sources was thus achieved using the blinking of the ring medianfiltered versions of the combined NACO images obtained in the $J, H$, and $K_{\mathrm{s}}$ bands. The availability of images obtained through several filters is a big advantage, as the fixed speckle cloud scales with the observation wavelength.

We derived aperture photometry for the detected sources using IRAF. We also attempted the PSF-fitting technique, but due to the large perturbations of the PSF shape by high spatialfrequency speckles close to the two stars, the result of the star subtraction was not satisfactory. We chose tight apertures of 24,12 , and 10 pixels in diameter $\left(0.32^{\prime \prime}, 0.16^{\prime \prime}\right.$, and $\left.0.13^{\prime \prime}\right)$, respectively for the $J, H$, and $K_{\mathrm{s}}$ bands, in order to reduce our sensitivity to the background fluctuations. By using such small apertures, we became more sensitive to the quality of the $\mathrm{AO}$ correction; but thanks to the brightness of the AO reference source ( $\alpha$ Cen B) and the generally good seeing, the Strehl ratio was relatively stable over our observations. The background level itself was estimated from the median flux of a ring of 50, 30 , and 20 pixels in diameter (respectively for $J, H$, and $K_{\mathrm{s}}$ ) and 10 pixels in thickness (in all cases).

The computation of the aperture correction was achieved on one of the brightest sources of our catalogue in the western field (star \#27 in Table 7). This source is located far enough (12") from $\alpha$ Cen B so that the local background can be considered 


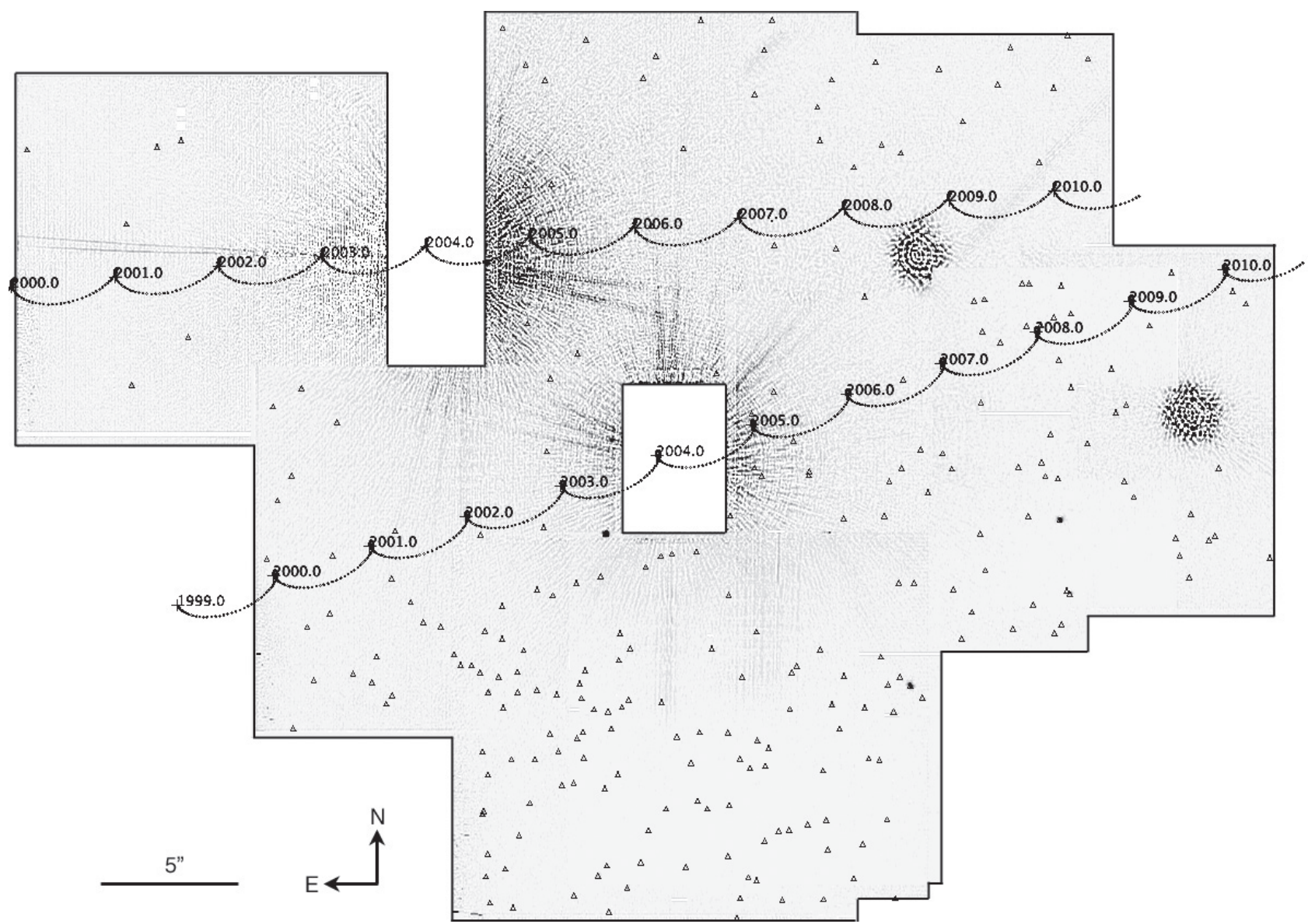

Fig. 4. Mosaic of the observed NACO fields of the environment of $\alpha$ Cen in the $K_{\mathrm{s}}$ band. This mosaic is a composite of the filtered versions of the original images, using the ring median filtering described in the text. The apparent ICRS positions on the sky of $\alpha$ Cen A and B, plotted for the 1999-2010 period, include their proper motion, orbital motion, and parallactic apparent displacement. The detected sources are represented as open triangles. The two circular features located to the west of the image are instrumental artefacts.

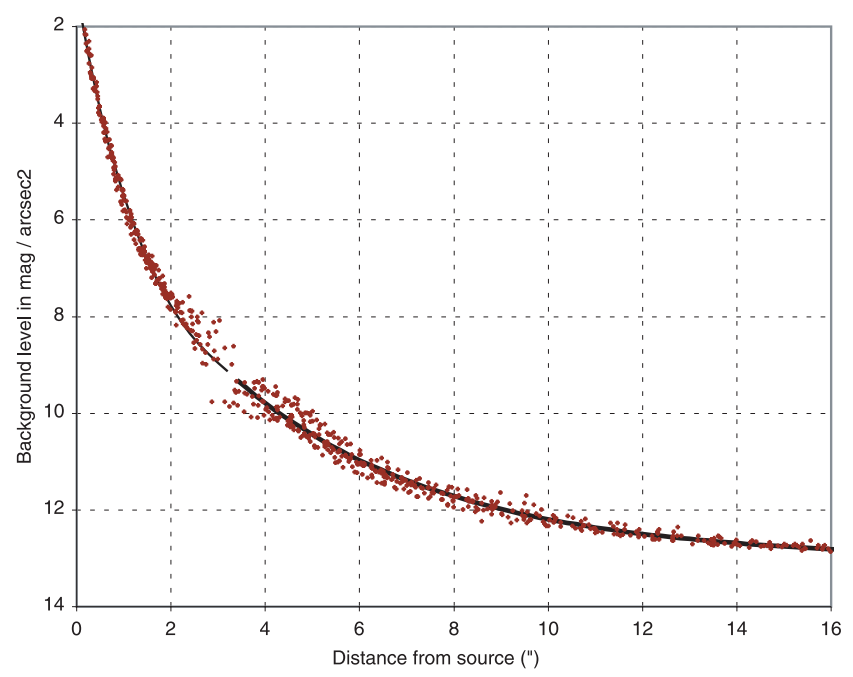

Fig. 5. Model fitting of the NACO diffuse background in the $K_{\mathrm{s}}$ band. The dots represent the individual measurements on the image, scaled to a zero-magnitude star, and the superimposed curves are the fitted models near to (thin curve) and far from (thick curve) the central star.

as flat. The resulting aperture correction of $\Delta m=1.0 \pm 0.2$, consistent in all three bands, was applied to the derived magnitudes. The photometric zero points were taken from ESO's routine instrument monitoring program. This is justified by the fact that the nights during which our observations were obtained were all of photometric quality. Considering the inhomogeneity of the background over which the photometry is measured, the contribution from the uncertainty to the photometric zero points is negligible. The airmass corrections were neglected: using the 2MASS values determined by Nikolaev et al. (2000; $A_{\mathrm{J}}=0.092$, $A_{\mathrm{H}}=0.031, A_{K}=0.065 \mathrm{mag} /$ airmass, relative to unity airmass), they are always smaller than 0.05 magnitudes in all three bands. In order to account for the Strehl ratio fluctuations, background inhomogeneity, and aperture correction uncertainty, a conservative systematic error was added to the statistical error bars listed in Table 7: \pm 0.8 mag on the $J$ band magnitudes (due to the relatively stronger diffused background) and \pm 0.5 mag on the $H$ and $K_{\mathrm{s}}$ magnitudes.

\subsection{Sensitivity}

The definition of the sensitivity of our search for companions around a binary star like $\alpha$ Cen is more difficult than for a single star. The presence of the combined diffused light from $\alpha$ Cen A and B in the NACO fields complicates the estimation of limiting magnitudes, as they become dependent on the position relative to the two bright stars.

We thus preferred to take advantage of the significant number of detected sources to derive a posteriori statistical properties and estimate the true sensitivity of our imaging survey. As shown in Fig. 6, the magnitudes of the faintest sources at 
Table 6. Diffused light model parameters. We considered an exponential model $\Delta m_{\lambda}=a \mathrm{e}^{b \theta}+c$, where $\Delta m_{\lambda}$ is the surface magnitude per $\operatorname{arcsec}^{2}$ of the sky background at an angular distance $\theta$ of a zero magnitude source, and $(a, b, c)$ are the adjusted model parameters.

\begin{tabular}{ccccc}
\hline \hline$\lambda$ & $J\left(\theta \geq 3^{\prime \prime}\right)$ & $H\left(\theta \geq 3^{\prime \prime}\right)$ & $K_{\mathrm{s}}\left(\theta \geq 3^{\prime \prime}\right)$ & $K_{\mathrm{s}}\left(\theta \leq 3^{\prime \prime}\right)$ \\
\hline$a$ & -7.281 & -7.969 & -8.035 & -9.068 \\
$b$ & -0.163 & -0.194 & -0.228 & -0.638 \\
$c$ & 13.226 & 13.247 & 13.018 & 10.305 \\
\hline
\end{tabular}
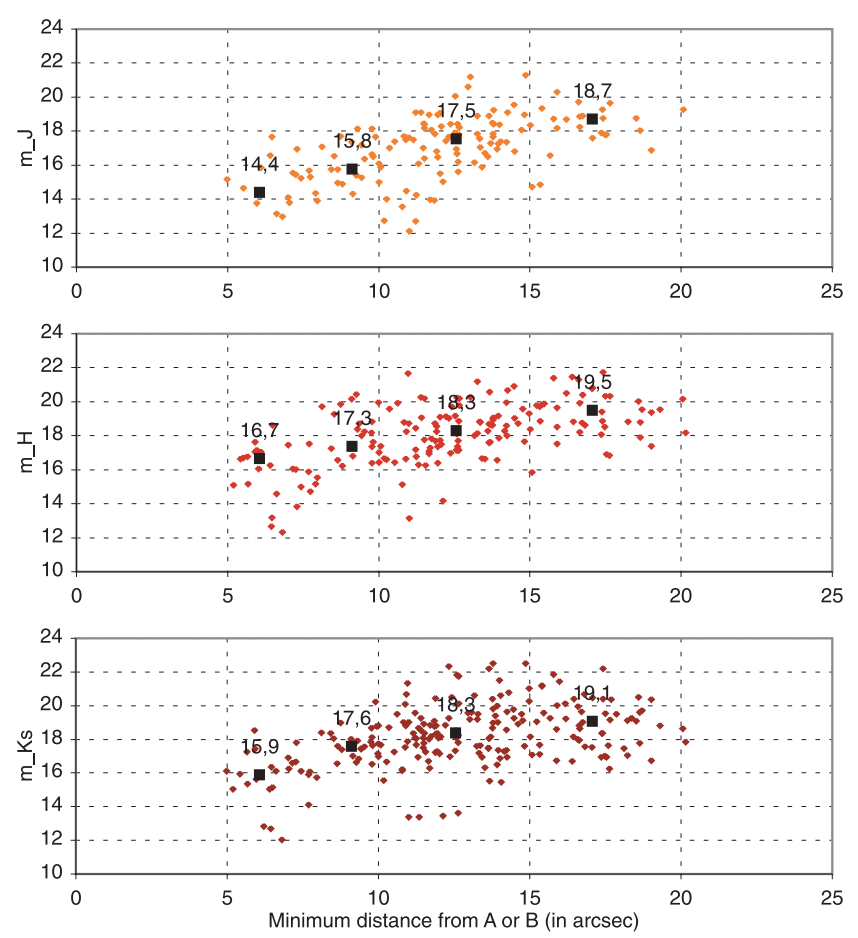

Fig. 6. Apparent magnitudes of the detected objects, as a function of their minimum angular separation with $\alpha$ Cen A or B. The solid squares correspond to the median magnitude of the objects detected in the 5-7", $7-10^{\prime \prime}, 10-15^{\prime \prime}$, and $15-20^{\prime \prime}$ domains, respectively. The corresponding magnitude is indicated in each case.

angular distances larger than $10^{\prime \prime}$ from A and B are $\approx 20$ in the $J$ band and $\approx 21$ in the $H$ and $K_{\mathrm{s}}$ bands. Note that several very faint and/or close-in sources, while they were clearly detected in the images, could not be measured by aperture photometry. To define our practical limiting magnitude, we chose to consider the median magnitude of the detected sources. This definition has the advantage of giving an empirical, statistically meaningful definition of the sensitivity, which can be expressed as a function of the distance to the two bright stars by computing the median within angular distance bins. Figure 6 shows the median of the detected object magnitudes in the $J, H$ and $K_{\mathrm{s}}$ bands for four angular distance bins: 6-7", 7-10", 10-15", and $\geq 15^{\prime \prime}$ as solid squares. The retained angular distance is the minimum of the source distances to A and B so as to account for the "saddle" shape of the diffused light from the two stars. In all bands, the limiting magnitudes at large angular distances are in the 18-20 range. They decrease to $m_{\mathrm{J}}=14.4, m_{\mathrm{H}}=16.7$, and $m_{K \mathrm{~s}}=15.9$ at $6^{\prime \prime}$.

We can compare these sensitivities with previous AO studies of the environment of bright stars. Using the same NACO instrument, Chauvin et al. (2005) obtained a depth of $m_{K \mathrm{~s}}=20$ around HIP $6856\left(m_{K \mathrm{~s}}=6.8\right)$ using an exposure of $10 \times 15 \mathrm{~s}$. With our typical exposures of $150 \times 3.5 \mathrm{~s}$, we are affected by additional readout noise, but the longer total exposure time compensates for this loss. On the bright single star Vega ( $m \approx 0$ in all bands), Metchev et al. (2003) used the PALAO system installed at the $5 \mathrm{~m}$ Hale reflector at Mount Palomar in the $J, H$ and $K_{\mathrm{s}}$ bands. With limiting magnitudes of $m_{\mathrm{H}} \approx 18$ at $20^{\prime \prime}$ and $\approx 16$ at $10^{\prime \prime}$, their study is slightly less sensitive than ours, but this can be explained by the smaller aperture. Macintosh et al. (2003) observed the same star using the Keck AO system and reach a deeper $m_{K \mathrm{~s}} \approx 20.5$ at $20^{\prime \prime}, 18.5$ at $10^{\prime \prime}$, and 17 at $7^{\prime \prime}$, using a $90 \times 15 \mathrm{~s}$ exposure. These figures are comparable to our results, although Vega is fainter by about $0.5 \mathrm{mag}$ than $\alpha$ Cen B in the infrared. From these comparisons, it appears that NACO is a well-suited instrument for studying the environment of bright stars, as its diffused light signature is relatively low (see also Sect. 3.4). In addition, the structure of the fixed-pattern speckle halo created by the monolithic primary mirror of the VLT-UT4 telescope appears smoother than with the Keck telescope's segmented primary mirror, thus making the identification of close companions easier.

\section{HST archive data}

As a complement to our NACO images, we searched the ESO/ST-ECF archive for images of $\alpha$ Cen. We subsequently analyzed the available data, that were obtained using three HST instruments: ACS, NICMOS and WFPC2. In this section, we present briefly our results.

\subsection{ACS}

A series of images was obtained centered on $\alpha$ Cen A star in September 2003 using the Advanced Camera for Surveys (ACS) onboard the Hubble Space Telescope, and these observations were repeated in January 2004 to check for the presence of proper motion companions. The same repeated series of images were obtained for $\alpha$ Cen B behind the coronagraphic mask in June 2004 and August 2004. In each case, eight images were recorded at eight wavelengths between $\lambda=754$ and $1024 \mathrm{~nm}$, with the FR914M broad ramp filter wheel (bandwidth of 9\%).

Using the coronagraphic mode of this instrument, the principle of the foreseen data analysis was to use the fact that the PSF of the instrument changes homothetically with the wavelength to remove most of the fixed-pattern speckle noise. As the position of the potential companions does not depend on the wavelength, their signature can be extracted more efficiently from the speckle noise than with a single image. This method is a particular application of the spectral deconvolution technique developed by Sparks \& Ford (2002). However, only one star of the $\alpha$ Cen pair at a time can be aligned with the coronagraphic spot. This results in a considerable amount of diffused light from the other, non-masked star, which also scales with the wavelength but with a different homothetic center. The application of the spectral deconvolution method is also made difficult by the slight undersampling of the PSF and by the availability of narrow-band filters instead of the continuous spectral coverage provided by a dispersive spectro-imaging instrument.

The pre-processing was achieved using the automated pipeline available at the HST archive. The images were subsequently co-added and filtered using the same procedure as the NACO images (Sect. 3.5). An extract of the $\alpha$ Cen B centered co-added coronagraphic image is presented in Fig. 3. Over a total field comparable to our NACO images, the number of objects 
detected in the ACS images is less than $10 \%$ of the NACO catalogue, corresponding to the brightest objects. We therefore limited our use of the ACS images to the definition of an accurate astrometric coordinate system (see Sect. 3.3).

\subsection{NICMOS}

The HST-NICMOS instrument (Thompson et al. 1998) is based on an infrared $\mathrm{HgCdTe} 256 \times 256$ pixel array sensitive over the $0.8-2.5 \mu \mathrm{m}$ range. Two series of exposures were taken through four filters on 19 October 1998 ( $\alpha$ Cen B) and 22 October 1998 ( $\alpha$ Cen A). The star images were positioned on the detector surface without a coronagraphic mask, producing heavy saturation within a radius of 2-3 arcsec around each star. The absence of a second observation makes it impossible to ascertain the comoving nature of potential companions. The complexity of the HST-NICMOS PSF (Krist et al. 1998) limits the sensitivity close to the star. Being too distant in time, there is no overlap between our NACO fields and these HST-NICMOS data. For these reasons, we decided not to include the NICMOS data in the present study.

\subsection{WFPC2}

The Wide Field and Planetary Camera 2 (WFPC2) is a twodimensional imaging photometer that covers the spectral range between 115 to $1050 \mathrm{~nm}$. Several accepted GTO and open time proposals, in particular by Ford et al. and Henry et al. in HST Cycles 4 to 7 resulted in a large amount of collected data. A total of 11 images centered on $\alpha$ Cen A were obtained in 1995 over two epochs (around May and August) in the $F 547 M, F 555 W, F 814 W$, and F850LP filters. In 1997, another series of 10 images was recorded, this time through the F953N and F1042M filters. The same sequences were also obtained with the WFPC2 field centered on $\alpha$ Cen B. As for the NICMOS data, these observations are too far in time from our NACO images, and there is almost no overlap between the fields. Therefore, we preferred not to include them in this study.

\section{Catalogue of the detected sources}

Table 7 lists the positions and $J H K_{\mathrm{s}}$ magnitudes of all the sources detected in the NACO images of $\alpha$ Cen. The right ascension $\alpha$ and declination $\delta$ refer to the ICRS and are not corrected for possible parallax. The epoch is J2004.5, corresponding to the mean observation time for stars observed in successive frames. As explained earlier, the typical positional uncertainty is not larger than 0.1 arcsec. The relatively high surface density of the detected objects can be explained by the fact that $\alpha$ Cen lies almost exactly in the Galactic plane and in a direction close to the Galactic center. This catalogue fills part of a long-standing "hole" in sky atlases, due to the diffused light from $\alpha$ Cen.

\section{Proper-motion companion search}

The very fast proper motion of $\alpha$ Cen should allow its comoving companions to be identified quickly. However, this is also a drawback due to the particularly dense star field around this binary star. The identification of the companion is not a trivial task because of the combination of the unknown orbital motion of the putative companion with the large proper motion and parallactic displacement of $\alpha$ Cen. Considering that $\alpha$ Cen moves an average of approximately one NACO pixel per day, the best strategy

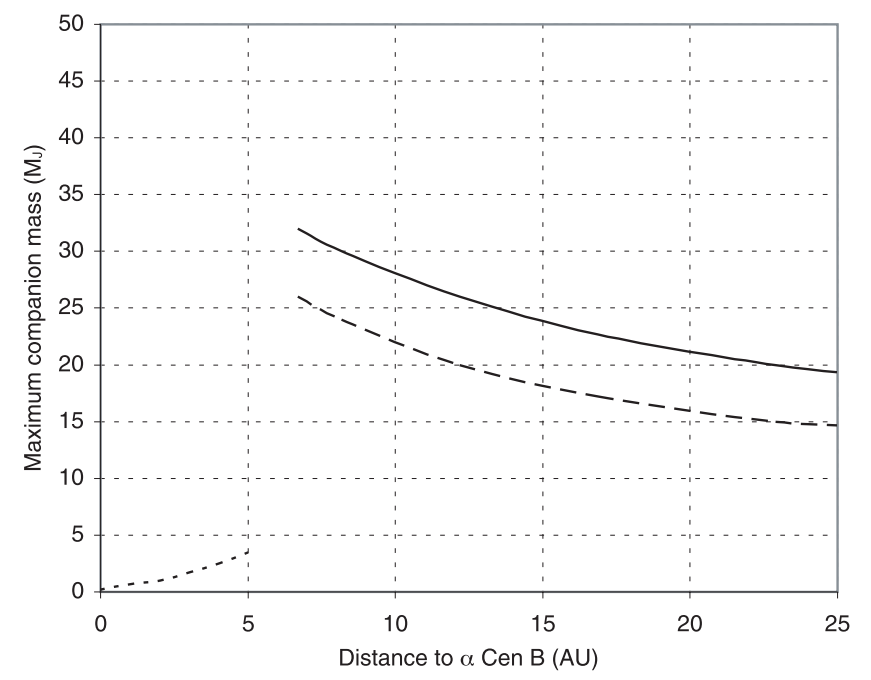

Fig. 7. Maximum mass of possible companions to $\alpha$ Cen B within the area explored by NACO imaging (encircled zone in Fig. 2, bottom). The solid curve corresponds to the median magnitude of the detected sources (Fig. 6, bottom) and the dashed line to the faintest detected objects. The dotted curve corresponds to the mass limit obtained by Endl et al. (2001) from radial velocity measurements.

would to observe the fields repeatedly with a time separation of 2 to 3 weeks. Unfortunately, due to scheduling constraints, our observations could not follow this scheme, and our first and second epochs were separated by about 10 months. The second and third series were separated by 5 months. Over these durations, the displacement of $\alpha$ Cen was considerable, resulting in a rather poor overlap of the different fields. Moreover, the diffused light from the two stars resulted in a moving zone of decreased sensitivity over part of the field.

In order to systematically search for statistically significant proper-motion companions, we applied the following procedure:

1. We converted the intensity images into SNR images, using the local background noise (mostly made of residual speckles). This allowed us to select only the sources that present an SNR of more than 3 per pixel, compared to the local noise, and a PSF shape (first a priori). For each epoch and each color, we therefore obtained a map of all point-like sources above the local noise.

2. The sources that could be identified at the same position on the sky at different epochs are background sources, so they were eliminated from our sample.

3. The second a priori knowledge that we can use is that any companion to $\alpha$ Cen will move on a Keplerian orbit. Therefore, its maximum orbital motion rate $\gamma$ is set by the third Kepler law and can be expressed as a function of the angular distance $\theta$ from the star:

$\gamma=\sqrt{\frac{G M_{*}}{\theta d^{3}}}$

where $d$ is the distance of the star, $M_{*}$ its mass, and $G$ the universal gravitational constant. A numerical application for $\alpha$ Cen results in the following maximum orbital motion:

$\gamma=\frac{3.83}{\sqrt{\theta}}$

where $\gamma$ is in $\operatorname{arcsec} / y r$ and $\theta$ in arcsec. Between two measurement epochs, we can therefore define a "possible 
orbital motion disk", centered on each identified point source, whose radius depends on the time lapse between the two epochs. The intersection of these disks with the list of identified sources in the following epoch allowed us to significantly reduce the number of candidate companions to just a few.

4. Eventually, the careful examination of the time evolution of the positions of the residual candidate companions allows the unphysical orbits to be rejected.

From this selection process, we could not identify any comoving companion within our overlapping regions. For only one source we were able to obtain a significant detection (more than $4 \sigma$ per pixel above the local noise) at epoch 2005.104, while no source was apparent at epoch 2004.137. Its ICRS coordinates are $\alpha=14: 39: 32.118 \delta=-60: 50: 08.60$, and its magnitude is estimated at $m_{K \mathrm{~s}}=17.7 \pm 0.5$. However, we could not identify any counterpart of this source within the "orbital circle" of angular radius $\gamma$, as defined in step 3 of our identification procedure. This source could be a distant variable star or a faint solar system object. We have not included it in the catalogue due to its unconfirmed nature.

\section{Discussion}

Massive substellar objects, as opposed to terrestrial planets, are detectable at very large distances from their parent star, as their magnitude is set by their intrinsic emission rather than by the reflected light. The age of the $\alpha$ Cen system is $5 \mathrm{Gyr}$, as determined by Thévenin et al. (2002) and confirmed by the interferometric diameters of the two stars (Kervella et al. 2003). Assuming a mass of $30 M_{\text {Jup }}$, a 5 Gyr-old giant planet has absolute magnitudes of $M_{H}=18$ and $M_{K}=20$, from evolutionary models by Baraffe et et al. (2003). At the distance of $\alpha$ Cen (1.3 pc), this translates into apparent magnitudes $m_{\mathrm{H}}=16$ and $m_{K}=18$, which were within reach of our NACO imaging search down to an angular separation of $\approx 5^{\prime \prime}$ from $\alpha$ Cen B (Fig. 6). Figure 7 gives the limiting sensitivity of our search in terms of companion mass, based on model magnitudes in the $K$ band from Baraffe et et al. (2003), and the $K_{\mathrm{s}}$ median magnitudes given in Fig. 6 (bottom). These are conservative estimates, considering that many sources that are fainter by up to two magnitudes have been detected in our images.

Murdoch et al. (1993) searched $\alpha$ Cen A and B for the radial velocity signature of BD companions with orbital periods $P \leq 4000$ days, but with a negative result. Using improved measurements obtained over a period of 5.5 years, Endl et al. (2001) concluded that no planet more massive than a few Jupiter masses, in projected $m \sin i$ value, is orbiting either $\alpha$ Cen A or B within 4 AU. If we follow the conclusions of Hale (1994) that the equatorial planes of A and B are probably coplanar with the binary orbit plane, and if we accept the hypothesis that exoplanets orbit in the equatorial plane of their parent star, then this projected mass value becomes a solid mass limit. The $J$ band search with the HST by Schroeder et al. (2000), which did not detect any companion, was limited to a sensitivity of $m_{J}=16$, corresponding to $40 M_{\mathrm{J}}$. Note however that the third star of the $\alpha$ Cen system Proxima probably does not host giant planets. This and other low mass stars were extensively scrutinized for any radial velocity variation, but did not show any (Kürster et al. 2003). Moreover, speckle-interferometry and imaging surveys (Leinert et al. 1997; Oppenheimer et al. 2001) failed to identify companions down to the BD masses around several low mass stars. Within our sensitivity and coverage limitations, our negative result leads toward the modeling results of Wiegert \& Holman (1997), who conclude that stable companion orbits may not exist beyond about $3 \mathrm{AU}$ from each component of the $\alpha$ Cen pair.

\section{Conclusion}

We have obtained deep adaptive-optics images of the close environment of $\alpha$ Cen A and B. From these images, we did not identify any comoving companion, but we assembled a catalogue of 252 faint background objects. Within the explored area, this negative result sets an upper mass limit of $20-30 M_{\mathrm{J}}$ to the possible substellar companions orbiting $\alpha$ Cen B. If companions of $\alpha$ Cen B exist, they are likely to orbit close to the star (within $5 \mathrm{AU}$ ) and to be less massive than a few times Jupiter (from radial velocity surveys). They could also be fainter than the current imaging search-limiting magnitude, but from Baraffe et al. (2003), a 5 Gyr-old, intermediate-mass exoplanet $\left(5 M_{\mathrm{J}}\right)$ around $\alpha$ Cen has an apparent magnitude of $m_{\mathrm{H}} \approx 27$, and $m_{K} \approx 39$, fully out of reach of the deepest imaging searches. Stars younger than $\alpha$ Cen could be more favorable targets, as the brightness of massive exoplanets is predicted to decrease steeply with time. However, the faintness of the detected background sources confirms the capabilities of modern adaptive optics instruments like NACO for exploring the close environment of very bright stars and for searching for massive exoplanets.

Acknowledgements. Based on observations made with the ESO Telescopes at Cerro Paranal Observatory under the Director's Discretionary Time (DDT) programs 272.C-5010, 273.C-5041 and 275.C-5027. We are grateful to ESO's Director General Dr. C. Cesarsky for this generous allocation of telescope time. We also wish to thank F. Namouni for fruitful discussions. This work made use of observations obtained with the NASA/ESA Hubble Space Telescope, obtained from the data archive at the Space Telescope Institute. STScI is operated by the Association of Universities for Research in Astronomy, Inc. under the NASA contract NAS 5-26555. This research made use of the SIMBAD and VIZIER databases at the CDS, Strasbourg (France), and of NASA's Astrophysics Data System Bibliographic Services.

\section{References}

Baraffe, I., Chabrier, G., Barman, T. S., Allard, F., \& Hauschildt, P. H. 2003, A\&A, 402, 701

Bigot, L., Kervella, P., Thévenin, F., \& Ségransan, D. 2006, A\&A, 446, 635 Chauvin, G., Lagrange, A.-M., Lacombe, F., et al. 2005, A\&A, 430, 1027

Cochran, W. D., Hatzes, A. P. Endl, M., et al. 2002, DPS, 34, 4202

Eggenberger, A., et al. 2003, in Scientific Frontiers in Research of Extrasolar Planets, ASP Conf., ed. Deming \& Seager

Eggenberger, A., Udry, S., \& Mayor, M. 2004, A\&A, 417, 353

Endl, M., Kürster, M., Els, S., Hatzes, A. P., \& Cochran, W. D. 2001, A\&A, 374, 675

ESA 1997, The Hipparcos and Tycho Catalogues, ESA SP-1200

Guenther, D. B., \& Demarque, P. 2000, ApJ, 531, 503

Hale, A. 1994, AJ, 107, 306

Holman, M., Touma, J., \& Tremaine, S. 1997, Nature, 386, 254

Innanen, K. A., Zheng, J. Q., Mikkola, S., et al. 1997, AJ, 113, 1915

Israelian, G., Santos, N., Mayor, M., \& Rebolo, R. 2004, A\&A, 414, 601

Kamper, K. W., \& Wesselink, A. J. 1978, AJ, 83, 1653

Kervella, P., Thévenin, F., Ségransan, D., et al. 2003, A\&A, 404, 1087

Konacki, M. 2005, Nature, 436, 230

Kozai, Y. 1962, AJ, 67, 591

Krist, J. E., Golimowski, D. A., Schroeder, D. J., \& Henry, T. 1998, PASP, 110, 1046

Kürster, M., Endl, M., Rouesnel, F., et al. 2003, A\&A, 403, 1077

Leinert, Ch., Henry, T., Glindemann, A., \& McCarthy, D. W. 1997, A\&A, 325, 159

Lenzen, R., Hofmann, R., Bizenberger, P., \& Tusche, A. 1998, Proc. SPIE 3354, 606

Macintosh, B. A., Becklin, E. E., Kaisler, D., Konopacky, Q., \& Zuckerman, B. 2003, ApJ, 594, 538

Martin, F., Conan, R., Tokovinin, A., et al. 2000, A\&AS Ser., 144, 39 
Masciadri, E., Brandner, W., Bouy, H., et al. 2003, A\&A, 411, 157

Mazeh, T., Krymolowski, Y., \& Rosenfeld, G. 1997, ApJ, 477, L103

Metchev, S. A., Hillenbrand, L. A., \& White, R. J. 2003, ApJ, 582, 1102

Morel, P., Provost, J., Lebreton, Y., Thévenin, F., \& Berthomieu, G. 2000, A\&A, 363,675

Mugrauer, M., Neuhauser, R., Seifahrt, A., Mazeh, T., \& Guenther, E. 2005, A\&A, 440, 1051

Murdoch, K. A., Hearnshaw, J. B., \& Clark, M. 11993, ApJ, 413, 349 (1993)

Nikolaev, S., Weinberg, M., Skrutskie, et al. 2000, AJ, 120, 3340

Oppenheimer, B. R., Golimowski, D. A., Kulkarni, S. R., et al. 2001, AJ, 121 , 2189

Pourbaix, D. 2000, A\&AS, 145, 215

Pourbaix, D., Nidever, D., McCarthy, C., et al. 2002, A\&A, 386, 280

Rouan, D., Riaud, P., Boccaletti, A., Clénet, Y., \& Labeyrie, A. 2000, PASP, 112 1479
Rousset, G., Lacombe, F., Puget, P., et al. 2000, Proc. SPIE, 4007, 72

Rousset, G., Lacombe, F., Puget, F., et al. 2003, Proc. SPIE, 4839, 140

Santos, N. C., Israelian, G., Mayor, M., Rebolo, R., \& Udry, S. 2003, A\&A, 398, 363

Sarazin, M., \& Roddier, F. 1990, A\&A, 227, 294

Schroeder, D. J., Golimowski, D. A., Brukardt, R. A., et al. 2000, AJ, 119, 906

Secker, J. 1995, PASP, 107, 496

Söderhjelm, S. 1999, A\&A, 341, 121

Sparks, W. B., \& Ford, H. 2002, ApJ, 578, 543

Thévenin, F., Provost, J., Morel, P., et al. 2002, A\&A, 392, L9

Thompson, R. I., Rieke, M., Schneider, G., Hines, D. C., \& Corbin, M. R. 1998, ApJ, 492, L95

Wiegert, P. A., \& Holman, M. 1997, AJ, 113, 1445 
P. Kervella et al.: Adaptive optics imaging of the environment of $\alpha$ Cen B, Online Material $p 1$

\section{Online Material}


P. Kervella et al.: Adaptive optics imaging of the environment of $\alpha$ Cen B, Online Material $p 2$

Table 7. Position and photometry of the sources detected around $\alpha$ Cen. The coordinates are for J2004.5 and refer to the ICRS.

\begin{tabular}{|c|c|c|c|c|c|}
\hline & $\alpha$ (h:m:s) & $\delta\left({ }^{\circ}:^{\prime}:^{\prime \prime}\right)$ & $m_{J}$ & $m_{H}$ & $m_{K \mathrm{~s}}$ \\
\hline 1 & $14: 39: 30.257$ & $-60: 50: 12.43$ & & $20.2 \pm 0.5$ & $18.6 \pm 0.5$ \\
\hline 2 & $14: 39: 30.375$ & $-60: 50: 03.19$ & & $19.4 \pm 0.7$ & $20.4 \pm 1.1$ \\
\hline 3 & $14: 39: 30.442$ & $-60: 50: 02.73$ & & $17.9 \pm 0.5$ & $19.6 \pm 0.6$ \\
\hline 4 & $14: 39: 30.511$ & $-60: 50: 09.16$ & & & $17.6 \pm 0.5$ \\
\hline 5 & $14: 39: 30.531$ & $-60: 50: 11.63$ & & & \pm 0.5 \\
\hline 6 & $14: 39: 30.560$ & $-60: 50: 11.80$ & & & $20.4 \pm 0.6$ \\
\hline 7 & $14: 39: 30.650$ & $-60: 50: 10.84$ & & $18.6 \pm 0.5$ & $17.6 \pm 0.5$ \\
\hline 8 & $14: 39: 30.658$ & $-60: 50: 13.15$ & & $19.4 \pm 0.5$ & $18.4 \pm 0.5$ \\
\hline 9 & 14:39:30.705 & $-60: 50: 12.36$ & & & $20.7 \pm 0.7$ \\
\hline 10 & $14: 39: 30.727$ & $-60: 50: 11.71$ & & & $20.4 \pm 0.6$ \\
\hline 11 & $14: 39: 30.747$ & $-60: 50: 02.05$ & & & $18.8 \pm 0.5$ \\
\hline 12 & $14: 39: 30.857$ & $-60: 50: 03.98$ & & $19.7 \pm 0.7$ & $17.7 \pm 0.5$ \\
\hline 13 & $14: 39: 30.935$ & $-60: 50: 10.22$ & & $18.7 \pm 0.5$ & $18.9 \pm 0.5$ \\
\hline 14 & $14: 39: 30.973$ & $-60: 50: 06.87$ & & & $15.5 \pm 0.5$ \\
\hline 15 & 14:39:30.982 & $-60: 50: 09.65$ & & $18.3 \pm 0.6$ & $17.3 \pm 0.5$ \\
\hline 16 & $14: 39: 31.024$ & $-60: 50: 07.15$ & & $17.6 \pm 0.5$ & $15.5 \pm 0.5$ \\
\hline 17 & $14: 39: 31.046$ & $-60: 50: 05.55$ & & $17.6 \pm 0.5$ & $17.5 \pm 0.5$ \\
\hline 18 & $14: 3$ & $-60: 4$ & & & $17.1 \pm 0.5$ \\
\hline 19 & $14: ?$ & $-60: 5$ & & .5 & $13.6 \pm 0.5$ \\
\hline 20 & $14: 3$ & $-60: 5$ & 18.3 & 17.5 & \pm 0.5 \\
\hline 21 & $14: 3$ & $-60: 5$ & 18.4 & $17.4 \pm 0.5$ & \pm 0.5 \\
\hline 22 & $14: 3$ & $-60: 5$ & $16.7=$ & $16.6 \pm 0.5$ & 0.5 \\
\hline 23 & $14: 3$ & $-60: 4$ & & & $19.2 \pm 0.5$ \\
\hline 24 & $14: 3$ & $-60: 50: 13.63$ & $17.1 \pm 0.8$ & $18.8 \pm 0.5$ & $17.6 \pm 0.5$ \\
\hline 25 & $14: 39: 31.296$ & $-60: 50: 14.85$ & $19.2 \pm$ & $19.1 \pm 0.5$ & $19.8 \pm 0.5$ \\
\hline 26 & $14: 39: 31.296$ & $-60: 50: 02.54$ & $16.2 \pm 0.8$ & $17.2 \pm 0.5$ & $18.2 \pm 0.5$ \\
\hline 27 & $14: 39: 31.304$ & $-60: 50: 11.06$ & $150+$ & $14.2 \pm 0.5$ & $13.4 \pm 0.5$ \\
\hline 28 & $14: 3$ & $-60: 5 c$ & $18.1 \pm 0.8$ & $17.9 \pm 0.5$ & $18.2 \pm 0.5$ \\
\hline 29 & $14: 39: 31.313$ & $-60: 50: 09.55$ & & $17.3 \pm 0.5$ & $16.3 \pm 0.5$ \\
\hline 30 & 14:39:31.331 & $-60: 50: 15.18$ & $18.5 \pm 0.8$ & $18.6 \pm 0.5$ & $18.7 \pm 0.5$ \\
\hline 31 & $14: 3$ & $-60: 5$ & $16.5 \pm 0.8$ & $16.4 \pm 0.5$ & \pm 0.5 \\
\hline 32 & 14: & $-60: 4$ & & & $=0.5$ \\
\hline 33 & & $-60:$ & $14.2 \pm 0.8$ & 0.5 & 0.5 \\
\hline 34 & & -60 & & & 0.5 \\
\hline 35 & 14:? & $-60: 5$ & $12.1 \pm 0.8$ & $13.1 \pm 0.5$ & 13 \\
\hline 36 & $14: 3$ & $-60: 5$ & & $19.1 \pm 0.5$ & 0.5 \\
\hline 37 & $14: 3$ & $-60: 4$ & & & \pm 1.6 \\
\hline 38 & $14: 35$ & $-60: 50: 14.15$ & $20.1 \pm 0.9$ & $18.3 \pm 0.5$ & $18.4 \pm 0.5$ \\
\hline 39 & $14: 39: 31.456$ & $-60: 50: 02.46$ & & $16.2 \pm 0.5$ & $17.3 \pm 0.5$ \\
\hline 40 & $14: 39: 31.461$ & $-60: 50: 10.91$ & & & $17.9 \pm 0.5$ \\
\hline 41 & $14: 39$ & $-60: 50: 04.00$ & 1 & 16 & \\
\hline 42 & $14: 39: 31.492$ & $-60: 50: 02.44$ & $16.1 \pm$ & $16.3 \pm 0.5$ & $18.7 \pm 0.5$ \\
\hline 43 & 14:39:31.509 & $-60: 50: 09.13$ & $12.7 \pm($ & $16.7 \pm 0.5$ & $15.6 \pm 0.5$ \\
\hline 44 & $14: 3$ & $-60: 50: 15.01$ & & $19.7 \pm 0.5$ & $20.5 \pm 0.5$ \\
\hline 45 & $14: 3$ & $-60: 49: 53.86$ & & & \\
\hline 46 & $14: 3$ & $-60: 50: 04.59$ & $16.7 \pm 0.8$ & $16.4 \pm 0.5$ & $16.5 \pm 0.5$ \\
\hline 47 & & $-60: 49: 55.21$ & & & \\
\hline 48 & & -60 & & & 0.5 \\
\hline 49 & & -60 & & 17 & \\
\hline 50 & & -60 & 15.4 & $20.4 \pm$ & 16 \\
\hline 51 & & -60 & $15.7 \pm$ & & 17. \\
\hline 52 & $14: 35$ & $-60: 50: 11.55$ & $16.4 \pm$ & $18.3 \pm 0.5$ & $17.6 \pm 0.5$ \\
\hline 53 & $14: 39: 31.730$ & $-60: 50: 03.09$ & $17.2 \pm$ & $18.0 \pm 0.5$ & $17.5 \pm 0.5$ \\
\hline 54 & .741 & $-60: 50: 14.40$ & $17.7 \pm 0.8$ & $18.8=$ & $19.0 \pm 0.5$ \\
\hline 55 & $14: 39: 31.788$ & $-60: 49: 5$ & & & \\
\hline 56 & $14: 39: 31.793$ & $-60: 50: 15.38$ & & 0.5 & \pm 0.5 \\
\hline 57 & $14: 39: 31.835$ & $-60: 50: 13.58$ & $18.1 \pm$ & $18.8 \pm 0.5$ & $18.7 \pm 0.5$ \\
\hline 58 & $14: 39: 31.842$ & $-60: 50: 09.19$ & $15.3 \pm$ & $14.7 \pm 0.5$ & $16.1 \pm 0.5$ \\
\hline 59 & 14:39:31.888 & $-60: 50: 08.63$ & $17.0 \pm 0.8$ & $13.8 \pm 0.5$ & $17.8 \pm 0.5$ \\
\hline 60 & 14:39:31.906 & $-60: 49: 54.64$ & & & \\
\hline 61 & $14: 39: 31.960$ & $-60: 50: 10.06$ & $15.6 \pm 0.8$ & $16.1 \pm 0.6$ & $=0.5$ \\
\hline 62 & $14: 39: 31.989$ & $-60: 50: 17.53$ & & & $18.1 \pm 0.5$ \\
\hline 63 & 14:39:31.997 & $-60: 50: 08.50$ & & & $16.4 \pm 0.5$ \\
\hline 64 & $14: 39: 32.029$ & $-60: 50: 13.35$ & $16.5 \pm 0.8$ & $19.3 \pm 0.6$ & $18.1 \pm 0.5$ \\
\hline 65 & 14:39:32.049 & $-60: 50: 17.08$ & & & $13.4 \pm 0.5$ \\
\hline 66 & $14: 3$ & $-60: 5$ & & 17 & $18.5 \pm 0.5$ \\
\hline 67 & $14: 39: 32.093$ & $-60: 50: 09.17$ & & $17.1 \pm 0.5$ & $17.4 \pm 0.5$ \\
\hline
\end{tabular}


P. Kervella et al.: Adaptive optics imaging of the environment of $\alpha$ Cen B, Online Material $p 3$

Table 7. continued.

\begin{tabular}{|c|c|c|c|c|c|}
\hline$\#$ & $\alpha$ (h:m:s) & $\delta\left({ }^{\circ}:^{\prime}:^{\prime \prime}\right)$ & $m_{J}$ & $m_{H}$ & $m_{K \mathrm{~s}}$ \\
\hline 68 & $14: 39: 32.095$ & $-60: 49: 57.70$ & & & $17.7 \pm 0.5$ \\
\hline 69 & $14: 39: 32.100$ & $-60: 50: 16.77$ & & & $20.1 \pm 0.7$ \\
\hline 70 & $14: 39: 32.105$ & $-60: 50: 13.34$ & $17.1 \pm 0.8$ & $19.7 \pm 0.6$ & $18.4 \pm 0.5$ \\
\hline 71 & $14: 39: 32.122$ & $-60: 50: 24.82$ & & & \\
\hline 72 & $14: 39: 32.132$ & $-60: 50: 18.03$ & & & $20.4 \pm 0.7$ \\
\hline 73 & $14: 39: 32.154$ & $-60: 50: 09.65$ & & $16.8 \pm 0.5$ & $17.3 \pm 0.5$ \\
\hline 74 & $14: 39: 32.160$ & $-60: 50: 17.06$ & & & $20.7 \pm 0.8$ \\
\hline 75 & $14: 39: 32.165$ & $-60: 50: 21.96$ & & & $21.2 \pm 0.6$ \\
\hline 76 & $14: 39: 32.178$ & $-60: 50: 10.91$ & $15.9 \pm 0.8$ & $17.0 \pm 0.5$ & $15.8 \pm 0.5$ \\
\hline 77 & $14: 39: 32.191$ & $-60: 49: 57.41$ & & & \\
\hline 78 & $14: 39: 32.196$ & $-60: 50: 16.04$ & $17.7 \pm 0.8$ & & $20.2 \pm 0.9$ \\
\hline 79 & $14: 39: 32.203$ & $-60: 50: 19.80$ & & & $19.5 \pm 0.5$ \\
\hline 80 & $14: 39: 32.222$ & $-60: 49: 54.39$ & & & $19.4 \pm 0.8$ \\
\hline 81 & $14: 39: 32.256$ & $-60: 50: 19.74$ & & & $19.6 \pm 0.5$ \\
\hline 82 & $14: 39: 32.276$ & $-60: 50: 17.89$ & & & $16.9 \pm 0.5$ \\
\hline 83 & $14: 39: 32.276$ & $-60: 50: 02.93$ & & & $12.8 \pm 0.5$ \\
\hline 84 & $14: 39: 32.285$ & $-60: 50: 22.85$ & & & $21.5 \pm 0.9$ \\
\hline 85 & $14: 39: 32.329$ & $-60: 49: 58.21$ & & & $18.8 \pm 0.5$ \\
\hline 86 & $14: 39: 32.330$ & $-60: 50: 24.11$ & & & \\
\hline 87 & $14: 39: 32.379$ & $-60: 50: 16.73$ & & & $18.8 \pm 0.5$ \\
\hline 88 & $14: 39: 32.381$ & $-60: 50: 10.99$ & $15.2 \pm 0.8$ & & $16.1 \pm 0.6$ \\
\hline 89 & $14: 39: 32.401$ & $-60: 50: 18.66$ & & & $18.9 \pm 0.5$ \\
\hline 90 & $14: 39: 32.419$ & $-60: 50: 01.17$ & $13.8 \pm 0.8$ & & $16.2 \pm 0.5$ \\
\hline 91 & $14: 39: 32.439$ & $-60: 50: 17.77$ & & & $16.1 \pm 0.5$ \\
\hline 92 & $14: 39: 32.441$ & $-60: 49: 55.03$ & $17.0 \pm 0.8$ & \pm 0.5 & $18.5 \pm 0.7$ \\
\hline 93 & $14: 3$ & $-60: 5$ & & 19 & $20.0 \pm 0.5$ \\
\hline 94 & $14: 39$ & $-60: 5$ & 19. & 17. & $17.6 \pm 0.5$ \\
\hline 95 & 14:3 & -60 & 19. & 20 & $19.2 \pm 0.5$ \\
\hline 96 & $14: 3$ & -60 & $21.2 \pm$ & $20.3 \pm$ & $19.2 \pm 0.5$ \\
\hline 97 & 14:39: & $-60: 5$ & $17.7 \pm 0.9$ & $19.9 \pm$ & $19.0 \pm 0.5$ \\
\hline 98 & $14: 39: 32.499$ & $-60: 49: 57.24$ & $17.4 \pm 0.9$ & $16.4 \pm$ & $17.3 \pm 0.5$ \\
\hline 99 & $14: 39: 32.512$ & $-60: 49: 56.03$ & & $16.9 \pm 0.5$ & $18.0 \pm 0.5$ \\
\hline 100 & $14: 39: 32.553$ & $-60: 50: 09.28$ & $13.4 \pm 0.8$ & $15.4 \pm 0.5$ & $17.4 \pm 0.9$ \\
\hline 101 & $14: 39: 32.555$ & $-60: 50: 09.43$ & & & \\
\hline 102 & $14: 39: 32.561$ & $-60: 50: 22.14$ & $21.3 \pm 1.1$ & & $22.5 \pm 0.9$ \\
\hline 103 & $14: 39: 32.615$ & $-60: 50: 16.36$ & $17.4 \pm 0.8$ & $20.2 \pm 0.9$ & $18.0 \pm 0.5$ \\
\hline 104 & $14: 39: 32.631$ & $-60: 50: 08.17$ & $12.9 \pm($ & $15.0 \pm$ & \\
\hline 105 & $14: 39: 32.640$ & 16.60 & $18.1 \pm 0.9$ & $18.4 \pm$ & 0.5 \\
\hline 106 & $14: 3$ & $-60:$ & & & \pm 0.5 \\
\hline 107 & & -60 & & 19. & $20.3 \pm 0.5$ \\
\hline 108 & 14 & -60 & & $20.3 \pm$ & $19.5 \pm 0.5$ \\
\hline 109 & $14: ?$ & -60 & & & $21.0 \pm 0.5$ \\
\hline 110 & 6 & $-60:$ & & 12 & $12.7 \pm 0.5$ \\
\hline 111 & $14: 3$ & -60 & $12.5 \pm 0.8$ & $14.9=$ & $15.4 \pm 0.5$ \\
\hline 112 & .738 & $-60: 4$ & & $17.7 \pm$ & $17.2 \pm 0.5$ \\
\hline 113 & $14: 39: 32.755$ & $-60: 50: 19.36$ & $19.0 \pm 0.8$ & $17.4 \pm 0.5$ & $17.3 \pm 0.5$ \\
\hline 114 & $14: 39: 32.771$ & $-60: 50: 20.01$ & & $20.2 \pm 0.6$ & $21.8 \pm 0.9$ \\
\hline 115 & $14: 39: 32.775$ & $-60: 50: 22.75$ & $14.9 \pm 0.8$ & $18.9 \pm 0.5$ & $17.1 \pm 0.5$ \\
\hline 116 & $14: 39: 32.777$ & $-60: 49: 53.95$ & & $21.7 \pm 0.9$ & $21.3 \pm 0.6$ \\
\hline 117 & $14: 39: 32.789$ & $-60: 50: 09.45$ & 1 & & \\
\hline 118 & $14: 3$ & $-60: 50: 19.09$ & $19.0 \pm 0.9$ & 17. & 0.5 \\
\hline 119 & & & & & \pm 0.5 \\
\hline 120 & & -6 & 15. & 5 & $15.9 \pm 0.5$ \\
\hline 121 & & -60 & $14.1 \pm 0.9$ & \pm 0.5 & $14.1 \pm 0.5$ \\
\hline 122 & & & & & $18.1 \pm 0.6$ \\
\hline 123 & & -60 & 1 & $14.1 \pm$ & \\
\hline 124 & & $-60: 5$ & & $19.8 \pm$ & 0.5 \\
\hline 125 & $14: ?$ & $-60:$ & $18.9 \pm 0$ & $18.2 \pm$ & $17.9 \pm 0.5$ \\
\hline 126 & $14: 39: 32.887$ & $-60: 50: 16.77$ & & $18.7 \pm$ & $16.8 \pm 0.5$ \\
\hline 127 & $14: 39: 32.896$ & $-60: 50: 24.83$ & $19.3 \pm 0.8$ & $18.9 \pm 0.5$ & $18.4 \pm 0.5$ \\
\hline 128 & $14: 39: 32.898$ & $-60: 50: 19.76$ & & & $22.3 \pm 1.7$ \\
\hline 129 & $14: 39: 32.913$ & $-60: 50: 25.54$ & & & $17.5 \pm 0.5$ \\
\hline 130 & $14: 39: 32.945$ & $-60: 50: 10.90$ & & $14.2 \pm 0.5$ & $11.8 \pm 0.5$ \\
\hline 131 & $14: 39: 32.951$ & $-60: 50: 21.44$ & & & $20.6 \pm 0.5$ \\
\hline 132 & $14: 39: 32.954$ & $-60: 50: 13.79$ & $16.6 \pm 0.9$ & $16.3 \pm 0.5$ & $15.1 \pm 0.5$ \\
\hline 133 & $14: 39: 32.958$ & $-60: 50: 23.30$ & $20.3 \pm 0.9$ & $18.5 \pm 0.5$ & $18.6 \pm 0.5$ \\
\hline 134 & $14: 39: 32.958$ & $-60: 50: 18.80$ & $19.1 \pm 1.0$ & $20.3 \pm 0.6$ & $19.3 \pm 0.5$ \\
\hline
\end{tabular}


P. Kervella et al.: Adaptive optics imaging of the environment of $\alpha$ Cen B, Online Material $p 4$

Table 7. continued.

\begin{tabular}{|c|c|c|c|c|c|}
\hline \# & $\alpha$ (h:m:s) & $\overline{\bar{\delta}\left({ }^{\circ}:^{\prime}::^{\prime \prime}\right)}$ & $m_{J}$ & $m_{H}$ & $m_{K \mathrm{~s}}$ \\
\hline 135 & $14: 39: 33.014$ & $-60: 50: 05.71$ & & & $14.1 \pm 0.5$ \\
\hline 136 & 14:39:33.038 & $-60: 50: 15.74$ & $15.8 \pm 0.8$ & $17.3 \pm 0.5$ & $18.4 \pm 0.5$ \\
\hline 137 & 14:39:33.061 & $-60: 50: 21.57$ & & & $18.6 \pm 0.5$ \\
\hline 138 & 14:39:33.092 & $-60: 49: 52.87$ & & $20.0 \pm 1.0$ & $17.2 \pm 0.5$ \\
\hline 139 & 14:39:33.099 & $-60: 50: 18.78$ & $16.4 \pm 0.8$ & & $18.9 \pm 0.5$ \\
\hline 140 & 14:39:33.109 & $-60: 50: 21.29$ & $18.4 \pm 0.8$ & & $21.5 \pm 0.8$ \\
\hline 141 & 14:39:33.114 & $-60: 50: 12.22$ & & 0.5 & $15.0 \pm 0.5$ \\
\hline 142 & 14:39:33.141 & $-60: 50: 19.90$ & $18.2 \pm 0.8$ & $20.2 \pm 0.7$ & $21.8 \pm 0.7$ \\
\hline 143 & 14:39:33.167 & $-60: 50: 23.69$ & & $18.8 \pm 0.5$ & $18.2 \pm 0.5$ \\
\hline 144 & 14:39:33.179 & $-60: 49: 57.53$ & & $18.6 \pm 2.1$ & $15.1 \pm 0.5$ \\
\hline 145 & $14: 3$ & $-60: 5$ & 13 & & $18.8 \pm 0.6$ \\
\hline 146 & 14:39:33.237 & $-60: 50: 12.29$ & & 15 & $15.4 \pm 0.5$ \\
\hline 147 & $14: 39$ & $-60: 50: 21.57$ & 1 & & $19.2 \pm 0.5$ \\
\hline 148 & $14: 39$ & 17.69 & & & $16.2 \pm 0.5$ \\
\hline 149 & $14: 39$ & $-60: 50: 12.37$ & $13.8 \pm 0.8$ & 17.2 & $15.6 \pm 0.5$ \\
\hline 150 & $14: 39$ & $-60: 49: 54.18$ & $14.3 \pm 0.8$ & 15.2 & \\
\hline 151 & 14:39: & $-60: 50: 00.36$ & $13.7 \pm 0.8$ & 15.4 & $18.6 \pm 1.0$ \\
\hline 152 & 3.353 & $-60: 50: 22.36$ & $19.3 \pm 0.8$ & 19.8 & $21.2 \pm 0.6$ \\
\hline 153 & $14: 39: 33.368$ & $-60: 50: 12.77$ & $13.2 \pm 0.8$ & $14.6 \pm 0.5$ & $16.1 \pm 0.5$ \\
\hline 154 & $14: 39: 33.379$ & $-60: 49: 54.96$ & $14.1 \pm 0.8$ & $17.4 \pm 0.5$ & $16.9 \pm 0.5$ \\
\hline 155 & 14:39:33.392 & $-60: 50: 23.52$ & $19.7 \pm 0.9$ & $21.3 \pm 0.7$ & $19.9 \pm 0.5$ \\
\hline 156 & 14:39:33.444 & $-60: 50: 15.73$ & $15.3 \pm 0.8$ & & $17.4 \pm 0.5$ \\
\hline 157 & 14:39:33.453 & $-60: 50: 17.61$ & $17.5 \pm 0.9$ & & $18.4 \pm 0.5$ \\
\hline 158 & 14:39:33.460 & $-60: 50: 24.45$ & & 16. & $16.3 \pm 0.5$ \\
\hline 159 & 14:39: & $-60: 50: 17.87$ & & & $17.5 \pm 0.5$ \\
\hline 160 & $14: 3$ & -60 & & & $17.0 \pm 0.5$ \\
\hline 161 & $14: 3$ & -60 & & 17. & $17.7 \pm 0.5$ \\
\hline 162 & 14: & -60 & & & $22.5 \pm 3.1$ \\
\hline 163 & 14 & -60 & & 17 & $17.0 \pm 0.5$ \\
\hline 164 & 14 & -60 & 18. & & $7.8 \pm 0.5$ \\
\hline 165 & 14: & -60 & $17.8 \pm 0.9$ & & $9.1 \pm 0.5$ \\
\hline 166 & .562 & $-60: 50: 23.63$ & & 20.8 & $19.1 \pm 0.5$ \\
\hline 167 & .567 & $-60: 50: 11.57$ & $13.0 \pm 0.8$ & $12.3=$ & $12.1 \pm 0.5$ \\
\hline 168 & $14: 3$ & $-60: 50: 20.84$ & $19.6 \pm 0.9$ & 20.9 & $19.8 \pm 0.5$ \\
\hline 169 & $14: 3$ & $-60: 50: 13.11$ & $13.9 \pm 0.8$ & 15.6 & $15.7 \pm 0.5$ \\
\hline 170 & 14: & $-60: 50: 24.01$ & $17.8 \pm 0.8$ & 16.9 & $16.9 \pm 0.5$ \\
\hline 171 & 14: & $-60:$ & $19.1 \pm 1.4$ & 19. & \\
\hline 172 & & -60 & & & 0.5 \\
\hline 173 & & -6 & & & 0.5 \\
\hline 174 & & -60 & & 19.0 & 0.5 \\
\hline 175 & & -60 & 20.6 & & $19.6 \pm 0.5$ \\
\hline 176 & & -60 & & & $18.3 \pm 0.5$ \\
\hline 177 & & -60 & & & $17.8 \pm 0.5$ \\
\hline 178 & 14 & -60 & & & $17.9 \pm 0.9$ \\
\hline 179 & 14 & -60 & & 21 & $19.2 \pm 0.5$ \\
\hline 180 & 3.713 & $-60: 50: 13.35$ & $14.9 \pm 0.8$ & $16.2 \pm 0.5$ & $17.4 \pm 0.5$ \\
\hline 181 & 3.725 & $-60: 50: 24.73$ & $18.8 \pm 0.8$ & & $19.1 \pm 0.5$ \\
\hline 182 & 3.727 & $-60: 50: 20.64$ & & 19 & $19.1 \pm 0.5$ \\
\hline 183 & $14: 3$ & $-60: 50: 20.70$ & 18 & $18.4 \pm 0.5$ & $18.2 \pm 0.5$ \\
\hline 184 & 14 & -60 & 17. & & \\
\hline 185 & 14: & 17.41 & $17.7 \pm 0.8$ & & 0.5 \\
\hline 186 & & -60 & & & 0.5 \\
\hline 187 & & -60 & $11.9 \pm 0.8$ & 13. & \\
\hline 188 & & $-60: 5$ & & $16.7 \pm 0.7$ & 0.5 \\
\hline 189 & & -60 & & & $22.2 \pm 1.9$ \\
\hline $0 \Omega$ & & -60 & & & \\
\hline 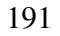 & & -60 & $14.6 \pm 0$ & & \\
\hline 192 & & -60 & & & .5 \\
\hline 193 & & -60 & $15.9 \pm 0.8$ & 17.4 & $17.7 \pm 0.5$ \\
\hline 194 & & -60 & $17.8 \pm 0.8$ & $18.9 \pm 0.5$ & $17.8 \pm 0.5$ \\
\hline 1 & & $-60: 50: 19.77$ & $18.1 \pm 0.8$ & & $19.5 \pm 0.5$ \\
\hline 196 & $14: 3$ & $-60: 50: 21.36$ & & & $20.1 \pm 0.5$ \\
\hline & $14: 3$ & $-60:$ & & & \\
\hline 198 & 14 & $-60: 4$ & & & \\
\hline 199 & $14: 3$ & -60 & & 137 & $14.2 \pm 0.5$ \\
\hline 200 & $14: 39$ & $-60: 50: 15.80$ & $16.8 \pm 0.8$ & $18.5 \pm 0.5$ & $18.8 \pm 0.5$ \\
\hline 201 & 14:39:33.999 & $-60: 50: 22.55$ & & $21.8 \pm 0.9$ & $20.4 \pm 0.5$ \\
\hline
\end{tabular}


P. Kervella et al.: Adaptive optics imaging of the environment of $\alpha$ Cen B, Online Material p 5

Table 7. continued.

\begin{tabular}{|c|c|c|c|c|c|}
\hline \# & $\alpha$ (h:m:s) & $\delta\left({ }^{\circ}:^{\prime}:^{\prime \prime}\right)$ & $m_{J}$ & $m_{H}$ & $m_{K \mathrm{~s}}$ \\
\hline 202 & $14: 39: 34.005$ & $-60: 50: 17.33$ & $16.2 \pm 0.8$ & $18.0 \pm 0.5$ & $20.6 \pm 0$ \\
\hline 3 & $14: 3$ & $-60: 50$ & $15.6 \pm 0.8$ & $17.7 \pm 0.5$ & $18.3 \pm 0.5$ \\
\hline 94 & $14: 39: 34.028$ & $-60: 50: 25.17$ & & & \\
\hline 205 & $14: 39: 34.037$ & $-60: 50: 19.77$ & & $10+8$ & $1 \pm 0$ \\
\hline 206 & $14: 39: 34.068$ & $-60: 50: 23.75$ & & $.5 \pm 0.5$ & $19.7 \pm 0.5$ \\
\hline 207 & 14:39:34.077 & $-60: 50: 17.89$ & $17.2 \pm 0.8$ & $19.2 \pm 0.5$ & $19.0 \pm 0.5$ \\
\hline 208 & $14: 39: 34.079$ & $-60: 49: 53.16$ & $15.2 \pm 0.8$ & $5.0 \pm 0.5$ & $16.1 \pm 0.5$ \\
\hline 209 & $14: 39: 34.083$ & $-60: 50: 15.38$ & $17.4 \pm 0.8$ & $19.1 \pm 0.5$ & $18.8 \pm 0.5$ \\
\hline 210 & $14: 39: 34.083$ & $-60: 50: 14.19$ & $17.0 \pm 0.8$ & & $8.1 \pm 0.5$ \\
\hline 211 & 14:39:34.101 & $-60: 50: 16.77$ & $17.9 \pm 0.8$ & $18.1 \pm 0.5$ & $17.6 \pm 0.5$ \\
\hline 212 & $14: 39: 34.144$ & $-60: 50: 24.99$ & $19.3 \pm 0.8$ & & \\
\hline 213 & 2 & $-60: 5$ & $17.0 \pm 0.8$ & 16 & $5 \pm 0.5$ \\
\hline 14 & & $-60:$ & $18.7=$ & .0 & $.2 \pm 0.5$ \\
\hline 15 & & -60 & & & $5 \pm 0.6$ \\
\hline 16 & & -60 & & & $8 \pm 0.5$ \\
\hline 7 & & -60 & $17.9 \pm 0.8$ & .5 & $3 \pm 0.5$ \\
\hline 18 & & -60 & .8 & $8.1 \pm 0.5$ & $0 \pm 0.5$ \\
\hline 219 & 14 & -60 : & $17.8 \pm 0.8$ & 18. & $0 \pm 0.5$ \\
\hline 20 & $14: 35$ & $-60: 50: 19.49$ & $16.6 \pm 0.8$ & & $19.3 \pm 0.5$ \\
\hline 21 & 14:39 & $-60: 5$ & $14.5 \pm 0.8$ & $18.3 \pm 0.8$ & $18.8 \pm 0.7$ \\
\hline 222 & $14: 3$ & $-60: 5$ & $18.9 \pm 0.8$ & $8.7 \pm 0.5$ & $17.4 \pm 0.5$ \\
\hline 223 & $14: 3$ & $-60: 5$ & $17.3 \pm 0.8$ & $18.7 \pm 0.5$ & $18.2 \pm 0.5$ \\
\hline 224 & $14: 39$ & $-60: 50: 16.34$ & & & $16.9 \pm 0.5$ \\
\hline 25 & 22 & $-60: 5$ & & 0.5 & $4 \pm 0.5$ \\
\hline 26 & & -60 & .8 & & \\
\hline & & & & & \pm 0.6 \\
\hline 28 & & -60 & & & \pm 0.5 \\
\hline 29 & & -60 & 15. & & $17.1 \pm 0.5$ \\
\hline 0 & & -60 & & .5 & 0.5 \\
\hline 31 & & -60 & & $20.6 \pm 1.5$ & 0.6 \\
\hline 2 & & -60 & $17.6 \pm 0.8$ & & 0.6 \\
\hline 3 & & -60 : & & 21 & $7 \pm 0.5$ \\
\hline 34 & $14: 3$ & $-60: 5$ & & $20.8 \pm 0.8$ & $17.5 \pm 0.5$ \\
\hline 35 & 14 & $-60: 50: 16.65$ & $18.7 \pm 0.9$ & & $22.2 \pm 1.4$ \\
\hline 36 & 14 & $-60: 50: 07.49$ & $15.0 \pm 0.8$ & 16 & $17.0 \pm 0.5$ \\
\hline 237 & & $-60:$ & & & $20.0 \pm 0.6$ \\
\hline 238 & & $-60: 5$ & 9 & & $21.9 \pm 1.2$ \\
\hline 239 & & $-60:$ & & & $19.1 \pm 0.5$ \\
\hline 240 & & -60 & & & \\
\hline 24 & & & \pm 0.8 & & 0.5 \\
\hline 2 & & -60 & & & $17.8 \pm 0.5$ \\
\hline 24 & & -60 & & & $18.1 \pm 0.5$ \\
\hline & & & & & \\
\hline & & & & & \\
\hline & & -60 & & & \\
\hline 247 & & $-60:$ & 17 & & \\
\hline 24 & & $-60: 4$ & 150 & $6.7 \pm 0.5$ & $17.0 \pm 0.5$ \\
\hline 249 & & $-60: 49: 57.48$ & & & $17.0 \pm 0.5$ \\
\hline & & $-60: 5$ & 3 & $17.5 \pm 0.5$ & $16.7 \pm 0.5$ \\
\hline 25 & & $-60: 5$ & $14.7 \pm$ & $15.9 \pm 0.5$ & $17.1 \pm 0.5$ \\
\hline 252 & $14: 39: 36.451$ & $-60: 49: 57.58$ & $16.9 \pm 0.8$ & $17.4 \pm 0.5$ & $16.7 \pm 0.5$ \\
\hline
\end{tabular}

